\title{
Estimation of the thermal diffusion coefficient in fusion plasmas taking frequency measurement uncertainties into account
}

\author{
M. van Berkel ${ }^{1,2,3}$, H. J. Zwart ${ }^{2 *}$, G. M. D. Hogeweij ${ }^{1}$, G. \\ Vandersteen $^{4}$, H. van den Brand ${ }^{1,2}$, M. R. de Baar ${ }^{1,2}$, and \\ the ASDEX Upgrade team \\ ${ }^{1}$ FOM Institute DIFFER-Dutch Institute for Fundamental Energy Research, \\ Association EURATOM- FOM, Trilateral Euregio Cluster, PO Box 1207, 3430 \\ BE Nieuwegein, The Netherlands \\ ${ }^{2}$ Eindhoven University of Technology, Dept. of Mechanical Engineering, Control \\ Systems Technology group (*Dynamics and Control group), PO Box 513, 5600 \\ MB Eindhoven, The Netherlands \\ ${ }^{3}$ National Institute for Fusion Science, 322 Oroshi-cho, Toki-city, Gifu, 509-5292, \\ Japan \\ ${ }^{4}$ Vrije Universiteit Brussel, Department of Fundamental Electricity and \\ Instrumentation, Pleinlaan 2, 1050 Brussels, Belgium \\ E-mail: m.v.berkel@tue.nl
}

\begin{abstract}
In this paper, the estimation of the thermal diffusivity from perturbative experiments in fusion plasmas is discussed. The measurements used to estimate the thermal diffusivity suffer from stochastic noise. Accurate estimation of the thermal diffusivity should take this into account. It will be shown that formulas found in the literature often result in a thermal diffusivity that has a bias (a difference between the estimated value and the actual value that remains even if more measurements are added) or have an unnecessarily large uncertainty. This will be shown by modeling a plasma using only diffusion as heat transport mechanism and measurement noise based on ASDEX Upgrade measurements. The Fourier coefficients of a temperature perturbation will exhibit noise from the Circular Complex Normal Distribution (CCND). Based on Fourier coefficients with a CCND, it is shown that the resulting Probability Density Function (PDF) of the thermal diffusivity is an inverse non-central chi-squared distribution. The thermal diffusivity that is found by sampling this distribution will always be biased and averaging of multiple estimated diffusivities will not necessarily improve the estimation. Confidence bounds are constructed to illustrate the uncertainty in diffusivity using several formulas that are equivalent in the noiseless case. Finally, a different method of averaging, that reduces the uncertainty significantly, is suggested. The methodology is also extended to the case where damping is included and it is explained how to include the cylindrical geometry.
\end{abstract}

Submitted to: Plasma Physics and Controlled Fusion 
Estimation of the thermal diffusion coefficient in fusion plasmas

\section{Introduction}

Perturbative experiments play an important role in the understanding and quantification of transport mechanisms in fusion reactors. In particular, the diffusivity of particle and thermal transport determine the efficiency of fusion reactors [1]. In practice, the observed thermal diffusivity differs one or two orders of magnitude from the neo-classical predictions. This difference is attributed to turbulent transport $[2,3]$. The detailed study of the thermal transport, in both neo-classical and turbulent regimes, requires reliable methods for measuring the thermal diffusivity including its confidence.

The analysis of perturbative experiments is used for studying various transport mechanisms in fusion plasmas $[4,5]$. Examples are the electron heat transport using Electron Cyclotron Resonance Heating (ECRH) [6, 7]; the momentum transport using modulated neutral beam injection to modulate the torque $[8,9,10]$; the ion heat transport using ion cyclotron resonance heating [11]; and the analysis of impurity transport $[12,13,14]$.

The method presented in this paper to arrive at reliable estimates for the diffusivity is in many cases applicable to the other transport channels as well. This certainly holds for the confidence analysis of amplitude and phase and if a number of additional assumptions are fulfilled also for the diffusivity (see Section 2.1). However, in this paper, we specifically analyze the electron thermal transport using locally deposited modulated ECRH. The ECRH induces perturbations in the electron temperature which are measured by Electron Cyclotron Emission (ECE) using a radiometer [15].

The study of the harmonic components of the perturbations induced by the modulated source at different radial locations gives information about the transport mechanisms, e.g. the perturbative electron heat diffusivity [16]. The perturbative electron diffusivity $\chi$ can be calculated based on the Fourier coefficients in terms of the phase and amplitude profiles. In this paper, the perturbative electron diffusivity is denoted as $\chi$ instead of $\chi_{e}^{H P}$, because the power balance electron heat diffusivity $\chi_{e}^{P B}$ determined in steady-state [4] is not analyzed here.

A number of important relationships are derived to determine the perturbative electron diffusivity $\chi$ on the basis of a single harmonic $\Omega$ either using the spatial phase derivative $\phi^{\prime}$, or the scaled amplitude derivative $A^{\prime} / A$, or a combination of these two in slab and cylindrical geometry using different assumptions on density gradients and non-diffusive contributions. They are summarized with their assumptions in $[4,5,17]$ and are used in many papers to characterize the electron thermal transport in tokamaks like JET [18, 19, 20], RTP [21, 22], ASDEX Upgrade [6, 23], DIII-D [24, 25], and TFTR [26]; and in stellarators like W7-AS [27] and LHD [28]; more recently perturbative experiments were used to determine the diffusion inside magnetic islands $[29,30]$. However, these relationships do not consider the uncertainty of the measurements leading to accuracy loss of the diffusion coefficient estimate. The reason for this loss in accuracy is the Probability Density Function (PDF) of $\chi$, which is directly associated with the method of calculation. This non-Gaussian PDF has a long tail, which has two important implications: 1) the estimated diffusion coefficients have a high upper uncertainty; 2) taking the average of estimated diffusion coefficients, e.g. for different harmonics, will result in a biased diffusivity estimate. Both implications will be demonstrated in this paper.

The standard assumption of Circular Complex Normal Distributed (CCND) noise 
on the Fourier coefficients is used. This distribution is the result of the Fourier transform of several different additive noise distributions including a Gaussian noise distribution. Based on the CCND and some minor conditions on the initial Signalto-Noise Ratio (SNR), it is shown that the resulting PDF of the diffusivity is an inverse non-central chi-squared-distribution. This PDF is derived via an analysis of the propagation of noise from the time domain to the estimated diffusion coefficient. This analysis also gives insight on how to arrive at the optimal diffusivity estimate by averaging the different harmonics, amplitude, and phase. A weighted averaging method using Maximum Likelihood Estimation is proposed [31]. This weighting is a sub-optimal solution in the sense that the resulting bias and variance on the diffusivity estimate is larger than the theoretically achievable bias and variance given the measurements. Nevertheless, it will still increase the accuracy significantly and gives a direct method for calculating the diffusivity. In case the damping is included, the product of $\phi^{\prime} A^{\prime} / A$ needs to be used. As no closed-form solution exists for this product of random Gaussian variables a Gaussian approximation is used based on recent work related to this topic [32]. This introduces some error, which can be studied using a Monte Carlo analysis.

The Cumulative Density Function (CDF) of $\chi$ is derived analytically for the case without damping and approximated in case of the damping. Hence, the accuracy of the estimated diffusion coefficients can be determined through the construction of confidence bounds.

This paper is structured as follows. Section 2 shortly introduces the commonly used relationships to calculate $\chi$ and their main assumptions. Moreover, it explains that Gaussian noise is the dominant noise on ECE-measurements. This allows for the derivation of the PDFs of amplitude and phase, which can be approximated under certain conditions by Gaussian distributions. This also holds for the PDFs of the phase derivative and the logarithmic amplitude derivatives, which are derived including their cross-correlation term. Section 3 uses these Gaussian approximations of the phase derivative and logarithmic amplitude derivative to determine the resulting PDFs of the diffusion coefficients. Its CDF is used to construct confidence bounds on the diffusivity. In addition, the PDF for $\chi$ is introduced based on the Gaussian approximation of the product of $\phi^{\prime} A^{\prime} / A$. Section 4 focuses on the practical aspects of estimating mean values and variances of the Fourier coefficients, where the variances refer to the variation of the Fourier coefficients with respect to their mean values. Direct methods for measuring the mean values and (co-)variances of the Fourier coefficients are presented, which are necessary to determine the PDFs of the diffusivity.

In Section 5 weighting methods are introduced to combine amplitude, phase, and different harmonics. These techniques are applied to simulations using realistic values for the Fourier coefficients and measurement noise extracted from ASDEX Upgrade data. Moreover, the influence of static errors, such as calibration errors is discussed. Finally, the main conclusions are summarized in Section 6.

\section{Distributions of phase and amplitude and its spatial derivatives}

The uncertainty on measurements can be quantified by studying the PDFs of the measurement data. First, it is motivated why the Gaussian distribution is the most probable noise distribution on our time domain measurements. This information is used to derive the PDFs of the Fourier coefficients, amplitude, and phase and their spatial derivatives. In addition, we show when the PDFs of amplitude and phase may 
be approximated by Gaussian distributions. However, first we shortly review the four common relationships to determine $\chi$ based on experimental data.

\subsection{Modeling of heat transport}

In this subsection the most common relationships used to calculate the perturbative heat diffusivity $\chi$ are summarized. These relationships will in this paper also be compared based on their statistical properties. This subsection is based on the work presented in $[17,33,34,35]$ and for the full details and derivations the reader is referred to [17]. All the relationships presented here assume that the transport coefficients are constant and that on the considered domain no source term is present. The relationships to calculate $\chi\left[-/ \mathrm{s}\right.$ or $\mathrm{m}^{2} / \mathrm{s}$ when rescaled in SI units] are based on a single harmonic $\Omega[\mathrm{rad} / \mathrm{s}]$ either using the spatial phase derivative $\phi^{\prime}[\mathrm{rad} / \mathrm{m}]$, or the scaled amplitude derivative $A^{\prime} / A[-/ \mathrm{m}]$, or a combination of these two. Basically, three cases have to be distinguished:

(i) The slab geometry case where both the convectivity and damping $\tau_{i n v}[\mathrm{~s}]$ $\left(\tau_{\text {inv }}=1 / \tau\right)$ are assumed zero resulting in

$$
\chi_{s 1}=\frac{3}{4} \frac{\Omega}{\phi^{\prime 2}}
$$

and

$$
\chi_{s 2}=\frac{3}{4} \frac{\Omega}{\left(A^{\prime} / A\right)^{2}} .
$$

Both imply that $A^{\prime} / A=\phi^{\prime}$. This can then also be rewritten by stating that $2 \phi^{\prime}=\phi^{\prime}+A^{\prime} / A$

$$
\chi_{s 3}=\frac{3 \Omega}{\left(A^{\prime} / A+\phi^{\prime}\right)^{2}},
$$

resulting in a third new relationship.

(ii) The slab geometry case where only the convectivity is assumed zero such that it accounts for the damping resulting in

$$
\chi_{s 4}=\frac{3}{4} \frac{\Omega}{\left(A^{\prime} / A\right) \phi^{\prime}} .
$$

This product is rewritten using the individual $\chi$ estimates based on (1) and (2) to calculate the diffusivity [5]

$$
\chi_{n}=\sqrt{\chi_{s 1} \chi_{s 2}},
$$

in which $\chi_{A}$ is the diffusivity calculated based on the amplitude and $\chi_{\phi}$ based on the phase. Both, these relationships can also be used to calculate $\chi$ when $\tau_{\text {inv }}=0$.

(iii) The cylindrical geometry case where also convectivity and damping are also included. In addition, also density gradients are included, which are assumed zero for the three other presented relationships. This relationship is derived in [17]

$$
\chi=\frac{3}{4} \Omega\left(\phi^{\prime}\left(\frac{A^{\prime}}{A}+\left\{\frac{1}{2 \rho}-\frac{n^{\prime}}{2 n}+\frac{U}{2 \chi}\right\}\right)+\frac{\phi^{\prime \prime}}{2}\right)^{-1},
$$

where $U$ is the convective velocity, $n$ the density and $n^{\prime}$ its spatial gradient. However, this relationship cannot be used in practice. Therefore, a number of 
assumptions and simplifying steps, such as assuming $U=0$ and replacing $\phi^{\prime \prime}$ with the derivative of $\phi^{\prime}$ from the slab-geometry are necessary to make it applicable in practice resulting in [17]

$$
\chi_{c}=\frac{3}{4} \Omega\left(\phi^{\prime}\left(\frac{A^{\prime}}{A}+\left\{\frac{1}{2 \rho}-\frac{n^{\prime}}{2 n}\right\}\right)\right)^{-1} .
$$

Interestingly, taking the spatial derivative of $\phi^{\prime}$ in slab geometry results in $\phi^{\prime \prime}$ to be zero. This implies that $\phi^{\prime}$ is linear such that between two different spatial locations

$$
\phi^{\prime}=\frac{\phi_{2}-\phi_{1}}{\Delta \rho},
$$

where $\Delta \rho=\rho_{2}-\rho_{1}$ and $\phi_{i}=\phi\left(\rho_{i}, \Omega_{k}\right) i=1,2$. It is formally proven in [36] that in slab-geometry under the assumption of constant parameter dependencies that $\phi^{\prime}$ is by definition given by (8) and under these assumptions the logarithmic amplitude derivative is given by

$$
\frac{A^{\prime}}{A}=\frac{d}{d \rho}(\ln (A))=\frac{\ln \left(A_{2}\right)-\ln \left(A_{1}\right)}{\Delta \rho} .
$$

In cylindrical geometry these relationships are no longer exact and then only approximate $A^{\prime} / A$ and $\phi^{\prime}$. Here, the derivative of amplitude and phase are based on two measurement points only. The reason is that under the assumptions used to derive (1), (2), and (4) the derivatives are defined by (8) and (9).

It is common practice to approximate $A^{\prime}$ and $\phi^{\prime}$ using interpolations of $A$ and $\phi$ or smoothed versions of $A$ and $\phi$. Here, we refrain from making such fits because: 1) they are unnecessary as the spatial derivatives are clearly defined and calculating $\chi$ and its confidence bounds and plotting at the different spatial locations still allows for the investigation of its spatial relationships; 2) in the slab geometry case such fits other than the one proposed here is inconsistent with assumptions under which the different relationships for $\chi$ have been derived [36]; 3) the results from such fits and the corresponding uncertainty will heavily depend on the chosen interpolation functions and their approximation order; 4) making such fits does not necessarily reduce the uncertainty and is rather a trade-off between spatial and temporal information; 5) after interpolation the new $A$ and $\phi$ have become spatially correlated due to the interpolation, which makes it more difficult to calculate the confidence bounds.

However, if one still prefers to use such fits, we suggest analyzing the uncertainty of the individual $A$ and $\phi$ measurement points. This uncertainty can be taken into account in the interpolation using some weighting to reduce the risk of mixing certain $A$ measurement points with uncertain $A$ measurement points. Then, when $A^{\prime}$ and $\phi^{\prime}$ have been determined with corresponding variance, the steps and the suggestions in the continuation of this paper can be used to combine the different harmonics and to determine the confidence bounds on $\chi$.

\subsection{Gaussian noise as the result of the central limit theorem}

In many applications the noise on measurements can be modeled using a Gaussian distribution function. This distribution is often the result of the central limit theorem, which states that, if many noise sources of different distributions are combined (convoluted), the resulting distribution tends towards a Gaussian distribution. More formal formulations of this theorem can be found in many statistics textbooks, e.g. 
[37]. This is the general argument for assuming a Gaussian distribution. However, for ECE measurements there is a stronger argument.

In this paper, ECE-measurements are used to determine the perturbative electron thermal diffusivity from the electron temperature perturbations. The dominant measurement noise on ECE-measurements is the thermal noise, which is generally Gaussian distributed [15]. More specifically, the thermal noise distribution on the output temperature measurements of the radiometer depends on the ratio between the intermediate frequency bandwidth $B_{I F}$ and the video bandwidth $B_{V}$. If $B_{I F} \gg B_{V}$, which holds for most radiometers used in fusion, the resulting distribution is Gaussian. This is theoretically derived in [38] and experimentally verified in [39].

\subsection{Normal complex circular distributed noise}

Assuming a Gaussian noise distribution in the time domain, the distribution functions of the Fourier coefficients can be determined. These distribution functions will be determined for every Fourier coefficient corresponding to a specific frequency $\Omega$.

The PDF of one Fourier coefficient at frequency $\Omega$ can be determined by taking the Fourier transform of a sinusoidal signal $T(t)$ with amplitude $M$ and phase $\theta$ and a Gaussian distributed additive noise term $e(t)$ with mean value zero and time domain variance $\sigma_{t}^{2}$

$$
T(t)=M \cos (\Omega t+\theta)+e(t) .
$$

The Fourier transform of (10) is not easily calculated. It requires the noise to be split in its harmonic components and the use of Hilbert transform properties. This transformation is described in [40,41] and is easily verified using a Monte Carlo analysis [42]. The Fourier coefficient $\Theta$ at frequency $\Omega$ has a bivariate distribution (PDF) in terms of its real part $\Theta_{\Re}$ and $\Theta_{\Im}$ imaginary part

$$
f_{\Theta}\left(\Theta_{\Re}, \Theta_{\Im}\right)=\frac{1}{2 \pi \sigma_{F}^{2}} \exp \left(-\frac{1}{2}\left(\frac{\Theta_{\Re}-\mu_{\Re}}{\sigma_{F}}\right)^{2}-\frac{1}{2}\left(\frac{\Theta_{\Im}-\mu_{\Im}}{\sigma_{F}}\right)^{2}\right) .
$$

The mean values of this distribution $\mu_{\Re}$ and $\mu_{\Im}$ can also be related to the mean value of the Fourier coefficient, i.e. $\hat{\Theta}=\mu_{\Re}+i \mu_{\Im}$. The variance $\sigma_{F}^{2}$ directly depends on $\sigma_{t}^{2}$, but also on the cross-correlation of the time domain noise. Therefore, instead of calculating $\sigma_{F}^{2}$ from $\sigma_{t}^{2}$, a different method is used, which directly estimates $\sigma_{F}^{2}\left(\Omega_{k}\right)$ from the measurements. This method is presented in Section 4.2.

The distribution $f_{\Theta(\Omega)}\left(\Theta_{\Re}, \Theta_{\Im}\right)$ is shown in Fig. 1 and is called a Circular Complex Normal Distribution (CCND). The real part $\Theta_{\Re}$ and imaginary part $\Theta_{\Im}$ are independently identically distributed (i.i.d.) and have a Gaussian distribution, see (11) or [40, 43]. It belongs to one Fourier coefficient at a specific frequency $\Omega$. This implies that for every Fourier coefficient $\Theta\left(\Omega_{k}\right)$, where $k$ denotes the excited harmonic, such a distribution can be defined, but with a different $\mu_{\Re}, \mu_{\Im}$, and $\sigma_{F}^{2}$. Moreover, the distribution of $\Theta\left(\Omega_{k}\right)$ is independent from the distribution of $\Theta\left(\Omega_{k+1}\right)$ [40, 43], which is important when different harmonics need to be combined.

The distribution $f_{\Theta}\left(\Theta_{\Re}, \Theta_{\Im}\right)$ can also be expressed in polar coordinates using the amplitude $A=\sqrt{\Theta_{\Re}^{2}+\Theta_{\Im}^{2}}$ and the phase $\phi$ defined as $\tan (\phi)=\Theta_{\Im} / \Theta_{\Re}$

$$
f_{A \phi}(A, \phi)=\frac{A}{2 \pi \sigma_{F}^{2}} \exp \left(-\frac{1}{2}\left(\frac{A \cos (\phi)-M \cos \theta}{\sigma_{F}}\right)^{2}\right) \cdot \exp \left(-\frac{1}{2}\left(\frac{A \sin (\phi)-M \sin \theta}{\sigma_{F}}\right)^{2}\right),
$$


(a) Scatter plot Fourier coeff.

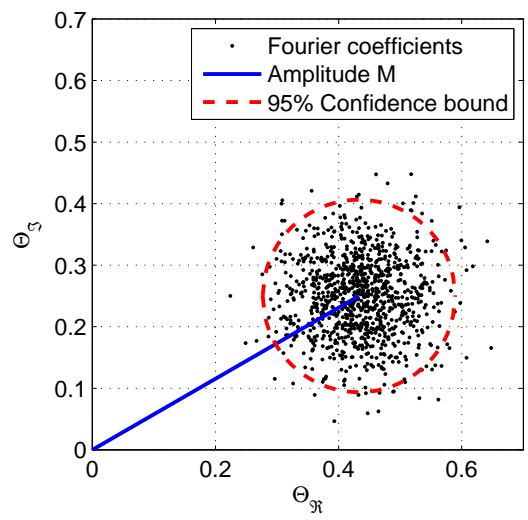

(b) Complex Circular Normal Distribution

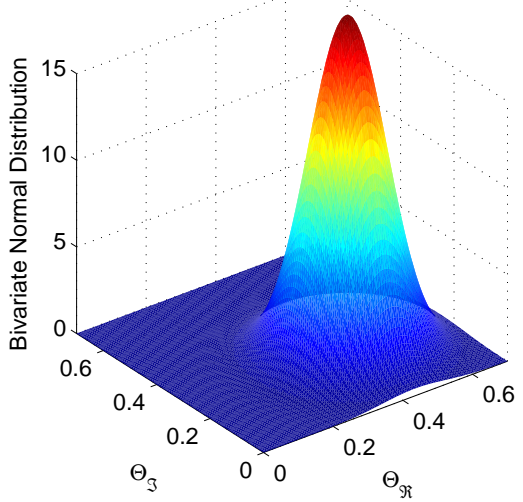

Figure 1. (a) A scatter plot of the Fourier coefficients calculated per period at frequency $\Omega$ of $x(t)=0.5 \sin \left(\Omega \omega t+\frac{\pi}{6}\right)+e(t)$, where $e(t)$ is Gaussian distributed noise. The Fourier coefficients are presented in the complex plane where $\Theta_{\Re}$ and $\Theta_{\Im}$ are its real and imaginary parts respectively. In addition, the $95 \%$ circular confidence bound is presented (red dashed). (b) The corresponding histogram/PDF of the Circular Complex Normal Distribution in the complex plane.

where $\mu_{\Re}=M \cos \theta$ and $\mu_{\Im}=M \sin (\theta)$ [41]. This form is more useful to calculate the PDFs of amplitude and phase, which will be necessary to determine the diffusivity. Finally, it is worth noting that the CCND is a good approximation of the Fourier transform for many other distributions in the time domain. However, whether the distribution is CCND depends on the number of time samples in the Fourier transform and a number of noise properties, which are not so easily derived [44, 43]. This can also be easily verified using Monte Carlo simulations. Therefore, in this paper rather than assuming a Gaussian distribution in the time domain, a CCND in the frequency domain is assumed. This extends the subsequent analysis to a much broader class of noise distributions in time domain.

\subsection{Amplitude and phase distributions and their confidence bounds}

The relationships introduced to determine the diffusion coefficient are based on the amplitude and phase of the measurements $[4,5]$. Therefore, the PDFs of the amplitude and phase are investigated. If the Signal-to-Noise ratio (SNR), defined here as $M / \sigma_{F}$, is large enough it can be shown that the PDFs of the phase and amplitude can be well approximated by a Gaussian distribution function.

The PDF of the amplitude can be found by integrating (12) over all the phases on a circle. The PDF of the phase can be found by integrating over all amplitudes on a line starting at the origin. The resulting PDF of the amplitude is the Rician distribution, which has two limit cases: the Rayleigh distribution when $M=0$ and the Gaussian distribution when $M / \sigma_{F} \longrightarrow \infty$ [41]. The resulting PDF of the phase is sometimes referred to as the Rician phase distribution [45] and is defined on the range $-\pi \leq \theta<\pi$. It has again two limit cases: the uniform distribution for $M=0$ and the Gaussian distribution for SNR $M / \sigma_{F} \gg 0$.

The evolution of the Rician distribution and Rician phase distribution for different 
(a) Rician Distributions (Amplitude)

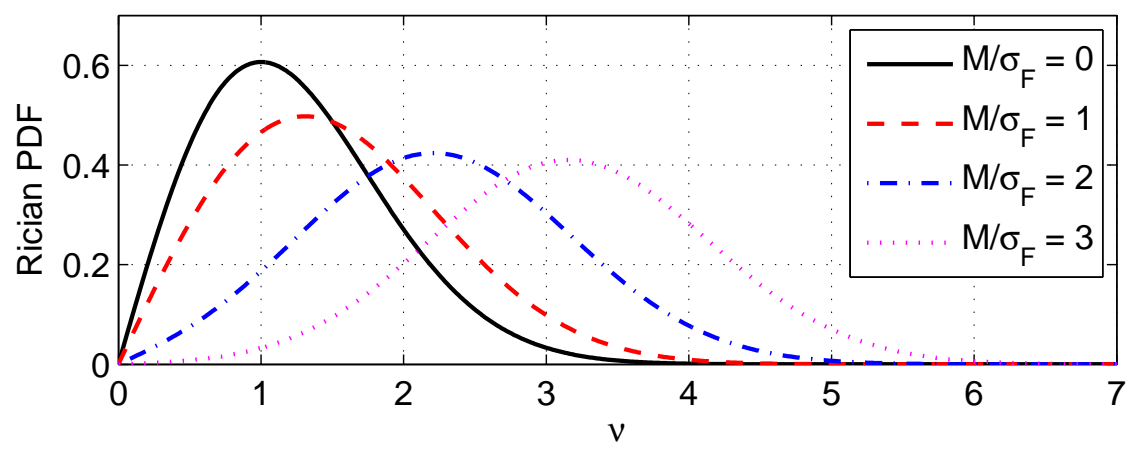

(b) Rician Phase Distributions (Phase)

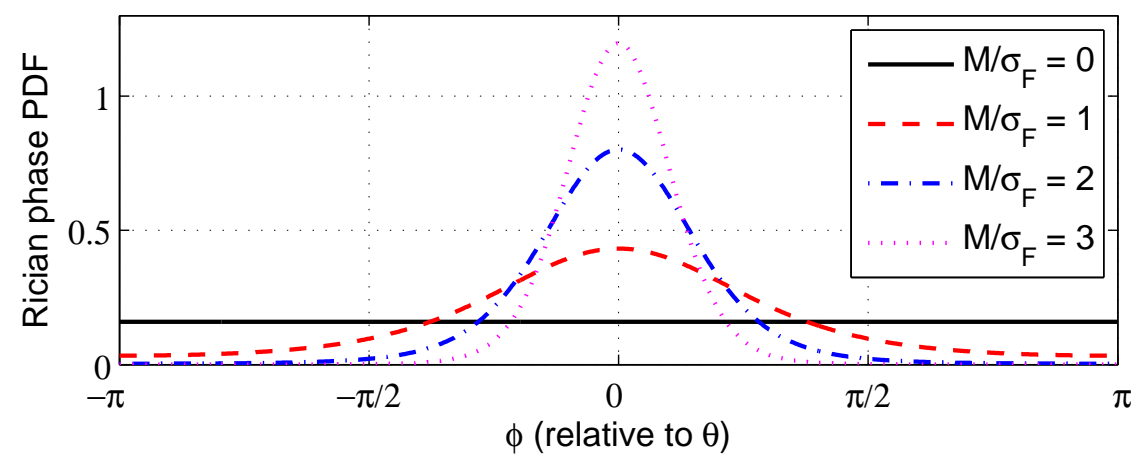

Figure 2. (a) Rician PDF of the amplitude for different values of the SNR using $\nu=A / \sigma_{F}$ as a scaling parameter. In the special case of $M / \sigma_{F}=0$, the Rician distribution becomes a Rayleigh distribution. (b) The Rician phase PDF for different values of the SNR. In the special case of $M / \sigma_{F}=0$, the Rician phase distribution reduces to a uniform distribution.

values of the $M / \sigma_{F}$ is presented in Fig. 2. It clearly shows that if $M / \sigma_{F}$ is large enough it can be well approximated by a Gaussian distribution function. Consequently, the mean value $\mu$ and the variance $\sigma^{2}$ of this Gaussian approximation need to be determined. The mean values of amplitude and phase are simply $\mu_{A}=M$ or $\mu_{\phi}=\theta$. However, the corresponding variances $\sigma_{A}^{2}$ and $\sigma_{\phi}^{2}$ need to be calculated using propagation of uncertainty, which is also called propagation of errors [31, 43]. It can be considered as a first order Taylor approximation for random variables around the mean value. In case of the phase this results in $\theta=\arctan \left(\mu_{\Im} / \mu_{\Re}\right)$

$$
\begin{aligned}
\sigma_{\phi}^{2} & =J_{\phi} \operatorname{cov}\left(\Theta_{\Re}, \Theta_{\Im}\right) J_{\phi}^{T} \text { with } \\
J_{\phi} & =\left[\frac{\partial \arctan \left(\mu_{\Im} / \mu_{\Re}\right)}{\partial \mu_{\Re}}, \frac{\partial \arctan \left(\mu_{\Im} / \mu_{\Re}\right)}{\partial \mu_{\Im}}\right],
\end{aligned}
$$

where $\operatorname{cov}\left(\Theta_{\Re}, \Theta_{\Im}\right)$ is a diagonal matrix with on the diagonal the variance of $\Theta_{\Re}$ and $\Theta_{\Im}$, i.e. $\sigma_{F}^{2}$, because $f_{\Theta(\Omega)}\left(\Theta_{\Re}, \Theta_{\Im}\right)$ is i.i.d. This results in the phase variance $\sigma_{\phi}^{2}=\sigma_{F}^{2} / M^{2}$. Similarly, also the variance of the amplitude can be calculated, which 
(a) Confidence bounds amplitude

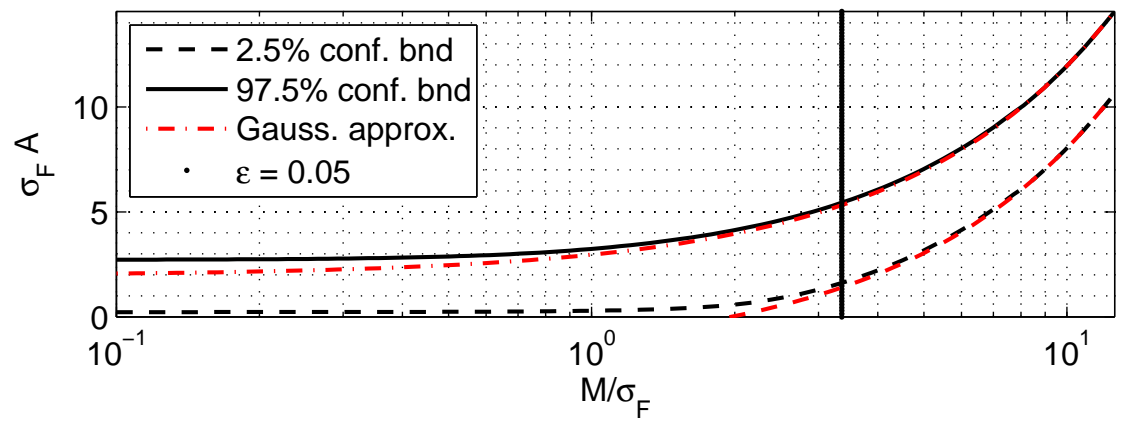

(b) Confidence bound phase

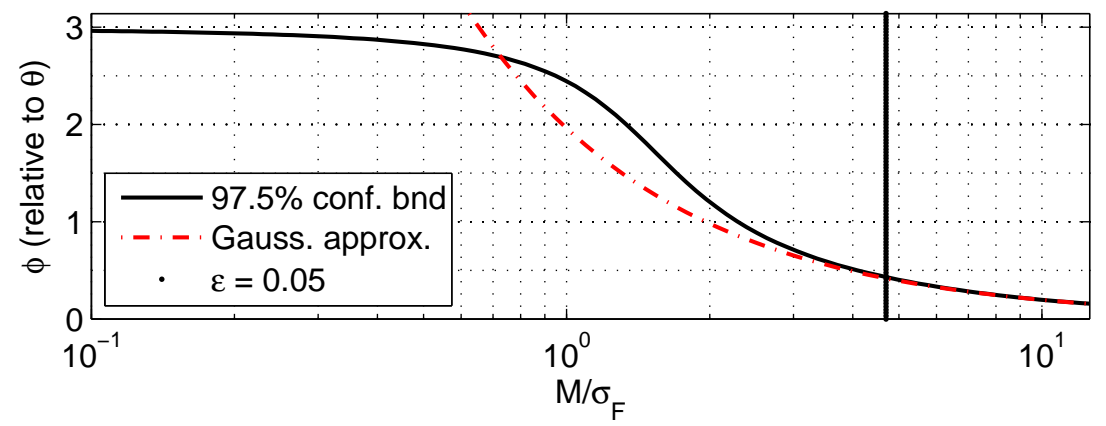

Figure 3. Comparison between the real Rician $95 \%$ confidence interval compared to the approximated confidence bound based on a Gaussian distribution. In addition, the vertical line (dotted line) represents the SNR where the relative error $\varepsilon=\left|\sigma_{\text {Rice }}^{2}-\sigma_{\text {Gauss }}^{2}\right| / \sigma_{\text {Gauss }}^{2}$ in terms of the variances equals $5 \%$. (a) The $2.5 \%$ (dashed line) and $97.5 \%$ (solid line) confidence bounds (95\% confidence interval) based on the Rician distribution function and the Gaussian approximations (red dashed-dotted line) of the confidence bounds for the $95 \%$ confidence interval. The y-axis is the amplitude $A$ scaled with $\sigma_{F}$. (b) the $97.5 \%$ confidence bound of the phase (solid line) based on the Rician phase distribution and the Gaussian approximation of the confidence bound (red dashed-dotted line). Note, that the Rician phase distribution is symmetric around $\phi$ (relative to $\theta$ ) such that one confidence bound suffices. The CDF to calculate the true confidence bounds can be found in Appendix A and in Appendix B.

is $\sigma_{A}^{2}=\sigma_{F}^{2}$. The corresponding confidence bounds for this Gaussian approximation are calculated as follows [31]

$$
C_{\phi}=\theta \pm \frac{\sigma_{F}}{M} \sqrt{2} \operatorname{erf}^{-1}(\mathfrak{p}) \text { or } C_{A}=M \pm \sigma_{F} \sqrt{2} \operatorname{erf}^{-1}(\mathfrak{p})
$$

in terms of a confidence $\mathfrak{p}$, e.g. $\mathfrak{p}=0.95$. However, these confidence bounds only hold for a significant SNR as is shown in Fig. 3, where also the true confidence bounds are shown. It shows that for a SNR $>5$, the Gaussian bound approximates the real confidence bounds well.

The Rician distribution is non-symmetric. This means that two confidence bounds are presented, i.e. $2.5 \%(\mathfrak{p}=0.025)$ and $97.5 \%(\mathfrak{p}=0.975)$ corresponding to $95 \%$ central confidence interval. In Appendix A and Appendix B, the derivation and calculation of the confidence bounds of amplitude and phase using Rician (phase) 
distributions are given, respectively.

The determination of $\chi$ requires the distributions of the scaled amplitude derivative $A^{\prime} / A$ and phase $\phi^{\prime}$ derivative, which are derived next.

\subsection{Distributions of $\phi^{\prime}$ and $A^{\prime} / A$}

In the previous section, it is shown that both amplitude and phase can be well approximated by a Gaussian distribution function. However, for the resulting PDFs of (8) and (9) this is not easily shown as it depends on five variables, which includes the mean values, the variances, and the cross-correlation of the noise between spatial locations $\rho_{i}$ and $\rho_{i+1}$. Therefore, here we simply approximate $\phi^{\prime}$ and $A^{\prime} / A$ using a Gaussian distribution function and assume that this is acceptable. This assumption is also supported by the fact that for the uncorrelated case (measurements at $\rho_{i}$ and $\rho_{i+1}$ are independent) it can be shown that for $\phi^{\prime}$ it is certainly Gaussian distributed remembering that a sum or difference of Gaussian variables results again in a Gaussian variable. In case of $A^{\prime} / A$ the analytic distribution function can be calculated assuming uncorrelated $A\left(\rho_{i}\right)$ and $A\left(\rho_{i+1}\right)$ (see Appendix C). Comparing, this distribution to its Gaussian approximation showed a good match for reasonable SNRs.

All the previous derivations and assumptions are related directly to the CCND, which is encountered often in practice even for non-Gaussian noise in time domain. Showing that it is reasonable to assume Gaussian distributed $A^{\prime} / A$ and $\phi^{\prime}$. However, here we wish to make the analysis more general. Therefore, also cross-correlation on the same harmonic (one spatial location) will be included and both $A^{\prime} / A$ and $\phi^{\prime}$ are directly approximated assuming jointly Gaussian distributions of the real and imaginary parts at spatial locations $\rho_{i}$ and $\rho_{i+1}$. This means that it is no longer assumed that the variances of real and imaginary parts are i.i.d., i.e. it is no longer assumed that $\sigma_{\Im}^{2}\left(\rho_{i}\right)=\sigma_{\Re}^{2}\left(\rho_{i}\right)$ and $\sigma_{\Re \Im}^{2}\left(\rho_{i}\right)=0$. The disadvantage of no longer assuming $\sigma_{\Im}^{2}\left(\rho_{i}\right)=\sigma_{\Re}^{2}\left(\rho_{i}\right)$ is that the confidence on these estimates decreases, because the real and imaginary are treated separately. However, both mean and variances can now by simply calculated assuming first order Taylor expansions.

The mean values are calculated using (9) and (8) such that

$$
\mu_{A^{\prime} / A}=\frac{1}{\Delta \rho} \ln \left(\frac{M\left(\rho_{i+1}\right)}{M\left(\rho_{i}\right)}\right) \text { and } \mu_{\phi^{\prime}}=\frac{\theta\left(\rho_{i+1}\right)-\theta\left(\rho_{i}\right)}{\Delta \rho} .
$$

Note, that it is important to unwrap the phase between spatial locations such that additional $2 \pi k$ rotations are avoided. The following symmetric covariance matrix is used to represent the (co-)variances $\left(\Omega_{k}\right.$ has been omitted)

$$
\begin{aligned}
& \operatorname{cov}\left(\Omega_{k}, \Theta\left(\rho_{i}\right), \Theta\left(\rho_{i+1}\right)\right)= \\
& {\left[\begin{array}{cccc}
\sigma_{\Re}^{2}\left(\rho_{i}\right) & \sigma_{\Re \Im}^{2}\left(\rho_{i}\right) & \sigma_{\Re \Re}^{2}\left(\rho_{i}, \rho_{i+1}\right) & \sigma_{\Re \Im}^{2}\left(\rho_{i}, \rho_{i+1}\right) \\
& \sigma_{\Im}^{2}\left(\rho_{i}\right) & \sigma_{\Re \Im}^{2}\left(\rho_{i}, \rho_{i+1}\right) & \sigma_{\Im \Im}^{2}\left(\rho_{i}, \rho_{i+1}\right) \\
& & \sigma_{\Re}^{2}\left(\rho_{i+1}\right) & \sigma_{\Re \Im}^{2}\left(\rho_{i+1}\right) \\
& & & \sigma_{\Im}^{2}\left(\rho_{i+1}\right)
\end{array}\right] .}
\end{aligned}
$$

The covariance matrix $\operatorname{cov}\left(\Omega_{k}, A^{\prime} / A, \phi^{\prime}\right)$ as function of $A^{\prime} / A$ and $\phi^{\prime}$ can be approximated using the first order Taylor approximation as follows

$$
\operatorname{cov}\left(\Omega_{k}, A^{\prime} / A, \phi^{\prime}\right)=J\left(\Omega_{k}\right) \operatorname{cov}\left(\Omega_{k}, \Theta\left(\rho_{i}\right), \Theta\left(\rho_{i+1}\right)\right) J^{T}\left(\Omega_{k}\right),
$$


where the Jacobian is given by

$$
J\left(\Omega_{k}\right)=\left[\begin{array}{cc}
-\frac{\mu_{\Re}\left(\rho_{i}\right)}{M^{2}\left(\rho_{i}\right) \Delta \rho} & \frac{\mu_{\Im}\left(\rho_{i}\right)}{M^{2}\left(\rho_{i}\right) \Delta \rho} \\
-\frac{\mu_{\Im}\left(\rho_{i}\right)}{M^{2}\left(\rho_{i}\right) \Delta \rho} & -\frac{\mu_{\Re}\left(\rho_{i}\right)}{M^{2}\left(\rho_{i}\right) \Delta \rho} \\
\frac{\mu_{\Re}\left(\rho_{i+1}\right)}{M^{2}\left(\rho_{i+1}\right) \Delta \rho} & -\frac{\mu_{\Im}\left(\rho_{i+1}\right)}{M^{2}\left(\rho_{i+1}\right) \Delta \rho} \\
\frac{\mu_{\Im}\left(\rho_{i+1}\right)}{M^{2}\left(\rho_{i+1}\right) \Delta \rho} & \frac{\mu_{\Re}\left(\rho_{i+1}\right)}{M^{2}\left(\rho_{i+1}\right) \Delta \rho}
\end{array}\right],
$$

which is based on the derivatives of (8) or (9) with respect to the real and imaginary parts, e.g. see (13). The resulting covariance matrix takes following form

$$
\operatorname{cov}\left(\Omega_{k}, A^{\prime} / A, \phi^{\prime}\right)=\left[\begin{array}{cc}
\sigma_{A^{\prime} / A}^{2} & \sigma_{A^{\prime} / A \phi^{\prime}}^{2} \\
\sigma_{\phi^{\prime} A^{\prime} / A}^{2} & \sigma_{\phi^{\prime}}^{2}
\end{array}\right]
$$

In case the confidence on (1) and (2) needs to be calculated the corresponding variances $\sigma_{\phi^{\prime}}^{2}$ and $\sigma_{A^{\prime} / A}^{2}$ can be extracted. In case, (4) and (7) are used then the full covariance matrix should be used.

The cross-correlation between $A^{\prime} / A$ and $\phi^{\prime}$, and two spatial locations in general, can be caused by for instance thermal noise inside the radiometer, which both measurement channels are subject to or it can be directly related to common temperature fluctuations in the plasma due to other sources (density fluctuation, ELMs, Sawteeth) than the perturbation source. In addition, as is shown in Fig. 1 and Fig. 3 the phase distribution directly depends on the amplitude $M$. This means that $A^{\prime} / A$ and $\phi^{\prime}$ are also correlated quantities by definition [45]. The advantage of using the (co-)variances is that it takes into account the uncertainty based on the measurements directly. However, this also means that the noise source cannot be distinguished unless the Fourier coefficients are again cross-correlated with the potential noise source.

Here, $A^{\prime} / A$ and $\phi^{\prime}$ are approximated by a Gaussian distribution function and using propagation of uncertainty its (co-)variances have been calculated. In the next section, based on the assumption of Gaussian distributed $A^{\prime} / A$ and $\phi^{\prime}$ the distribution of $\chi$ and its confidence bounds are calculated for the relationships presented in Section 2.1 .

\section{Distributions of the diffusivity $\chi$}

In this section, we derive the distribution functions of $\chi$ for the relationships given in Section 2.1 to determine the confidence bounds on $\chi$. It is separated in three parts: 1) the PDF for $\chi$ based on $\left(A^{\prime} / A\right)^{2}$ and $\left(\phi^{\prime}\right)^{2}$ are calculated, which corresponds to the case where damping is zero; 2 ) its corresponding CDF is calculated allowing for the calculation of the confidence bounds for $\chi ; 3)$ the PDF of $\chi$ based on a Gaussian approximation of $\phi^{\prime} A^{\prime} / A$ corresponding to the case where the damping can also be non-zero.

\subsection{Inverse non-central chi-squared distribution}

In the previous sections, we have shown that the distribution functions of $\phi^{\prime}$ and $A^{\prime} / A$ can be well-approximated by Gaussian distribution functions. This is also true for the distribution of the diffusivity, but only for very small noise variances, which in practice are rarely encountered, especially when considering higher harmonics. The reason is that the diffusivity distribution is the squared reciprocal of the derivatives of 
the phase or the amplitude, which results in a very different distribution. Here, only the distributions for (1) and (2) are discussed.

The derivation of the PDF of $\chi$ can be simplified by introducing a variable $\gamma$, where $\gamma$ denotes either $A^{\prime} / A$ or $\phi^{\prime}$. The PDF of $\gamma$ is then denoted by $g(\gamma)$. This, $g(\gamma)$ is transformed to $h(\chi)$, the PDF of $\chi$, using conservation of area. Then, it follows from (1) or (2) that $\gamma=\sqrt{3 / 4 \cdot \Omega / \chi}$ and that the derivative $|d \chi / d \gamma|^{-1}=\sqrt{3 / 16 \cdot \Omega / \chi^{3}}$. Hence, the resulting distribution function of $\chi$ is given by

$$
h(\chi\{\gamma\})= \begin{cases}\sqrt{\frac{3}{16} \frac{\Omega}{\chi^{3}}}\{g(\gamma)+g(-\gamma)\} & \chi>0 \\ 0 & \chi \leq 0\end{cases}
$$

If a Gaussian approximation of $g\left(\frac{A^{\prime}}{A}\right)$ or $g\left(\phi^{\prime}\right)$ is used, then

$$
g(\gamma)=\frac{1}{\sqrt{2 \pi \sigma_{\gamma}^{2}}} \exp \left(-\frac{\left(\gamma-\mu_{\gamma}\right)^{2}}{2 \sigma_{\gamma}^{2}}\right)
$$

and $h(\chi)$ is a special case of the inverse non-central chi-squared distribution. This distribution is positive non-symmetric with a large (right) tail and only resembles a Gaussian for small variances. In Fig. 4 the inverse non-central chi-squared distribution, $h(\chi)$ is shown. It clearly shows that $h(\chi)$ has a long tail especially when the variance $\sigma_{\gamma}^{2}$ is large. The long tail also results in a bias, which is defined as the difference between the expected value $\mathbb{E}\{\chi\}$ and the true value of $\chi$. However, it is difficult to quantify this bias, because the expected value $\mathbb{E}\{\chi\}$, defined by the improper integral

$$
\mathbb{E}\{\chi\}=\int_{0}^{\infty} \chi h(\chi) d \chi=\infty
$$

is divergent. This has important implications, because it means that if the diffusivity is determined a number of times from an experiment with the same $\mu_{\gamma}$ and $\sigma_{\gamma}^{2}$, the average of these experiments will not result in the true diffusivity $\chi$, i.e. it will be biased. Even worse, the diffusivity estimate will diverge to $\infty$ for an increasing number of estimates and its divergence rate depends on the variance $\sigma_{\gamma}^{2}$.

\subsection{Confidence bounds non-central inverse chi-squared distribution}

The confidence bounds on the diffusivity can now also be calculated based on (20) by calculating its Cumulative Density Function (CDF). The CDF $H(X)$ of the PDF $h(\chi)$ is given by $H(X)=\int_{0}^{X} h(\chi) d \chi$, which can be solved analytically

$$
H(X)= \begin{cases}1-\frac{1}{2} \operatorname{erf}\left(\frac{\sqrt{\frac{3}{4} \frac{\Omega}{X}}+\mu_{\gamma}}{\sigma_{\gamma} \sqrt{2}}\right)-\frac{1}{2} \operatorname{erf}\left(\frac{\sqrt{\frac{3}{4} \frac{\Omega}{X}}-\mu_{\gamma}}{\sigma_{\gamma} \sqrt{2}}\right) & X>0 \\ 0 & X \leq 0\end{cases}
$$

The CDF $H(X)$ is non-symmetric, which means that two confidence bounds need to be calculated. We are interested in the central confidence interval such that the lower bound $X_{\min }$ is determined by $H\left(X_{\min }\right)=\frac{1-\mathrm{p}}{2}$ and the upper bound by $H\left(X_{\max }\right)=\frac{1+\mathfrak{p}}{2}$. The CDF and the corresponding $\mathfrak{p}=0.95$ central confidence interval is shown in Fig. 4.

In practice, $H(X)$ is difficult to invert analytically. On the other hand, the bounds can be easily calculated by finding the zero crossing of $H(X)-\frac{1-\mathfrak{p}}{2}$ and $H(X)-\frac{1+\mathfrak{p}}{2}$, for which many algorithms exist. 
(a)

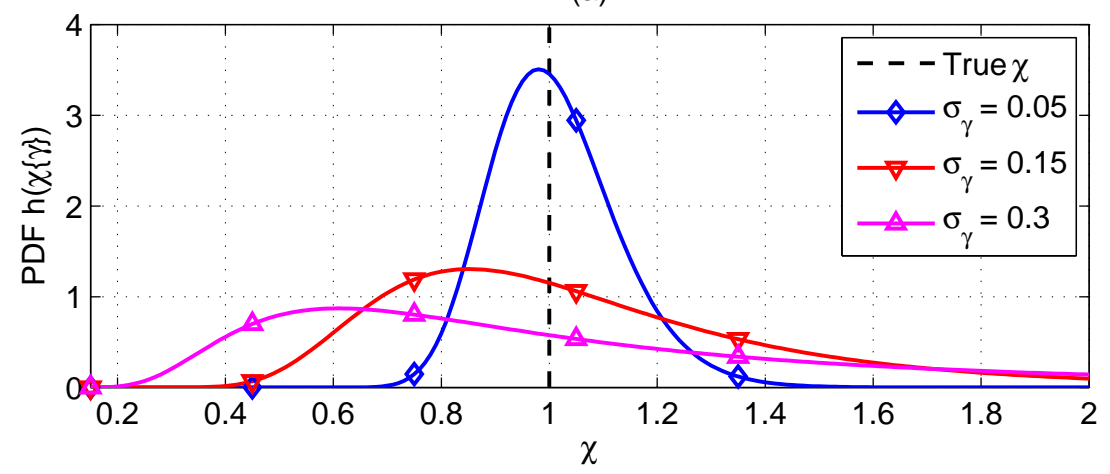

(b)

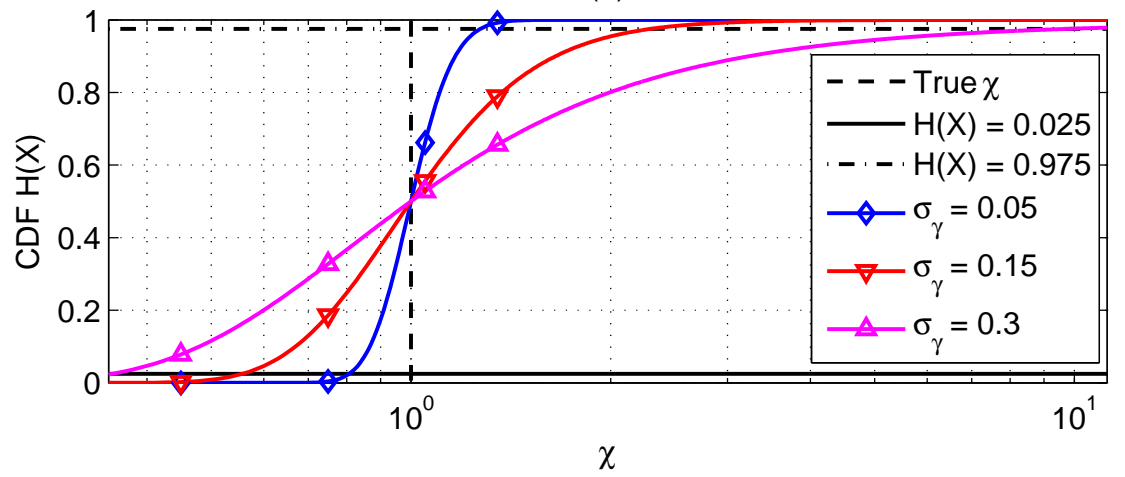

Figure 4. (a) The inverse non-central chi-squared distribution for different values of the variance $\sigma_{\gamma}^{2}, \mu_{\gamma}=0.866$ and $\Omega=1$, which corresponds to $\chi=1$ (represented by the dashed line). (b) The Cumulative Density Function $H(X)$ of the inverse non-central chi-squared distribution corresponding to the presented PDFs. The dashed line represents the value of $\chi$ when $\sigma_{\gamma}^{2} \rightarrow 0$. The solid line show the values at which $H(X)=0.025$ and the dashed-dotted line where $H(X)=0.975$ corresponding to a central $95 \%$ confidence interval.

\subsection{Inverse product distribution function}

The product of the Gaussian distributed variables $\phi^{\prime}$ and $A^{\prime} / A$ plays an important role in (4) and (6) in which the effect of damping is suppressed. The distribution for $\chi(20)$ is a special case of the distribution discussed here as it assumes that $\mu_{\phi^{\prime}}=\mu_{A^{\prime} / A}$ and $\sigma_{A^{\prime} / A}^{2}=\sigma_{\phi^{\prime}}^{2}$ as such it is a simplification of the general product of $\phi^{\prime}$ and $A^{\prime} / A$. The distribution function of the general product of $\phi^{\prime}$ and $A^{\prime} / A$ is treated here separately, because it does not have a closed-form solution [46]. This also holds for the PDF of $\chi$ based on (4). This complicates the calculation of the confidence bounds significantly, because the CDF needs to be solved using a double integral.

As the closed-form solution does not exist other approaches are necessary. Therefore, in the literature the distribution function is generally approximated using various distribution functions for the different limit cases [47]. In case the ratio's $\mu_{A^{\prime} / A} / \sigma_{A^{\prime} / A}$ and $\mu_{\phi^{\prime}} / \sigma_{\phi^{\prime}}$ are large then the product can be approximated well by a Gaussian distribution [48]. Unfortunately, the ratio's in practice acquired in this 
paper do not all satisfy the requirement on these ratio's. On the other hand, using different approximations are impractical due to their complexity. This especially holds for optimally combining different measurements (harmonics), which is well defined for Gaussian distributed variables, but not for many of the other proposed distribution functions. Therefore, here is chosen to approximate the product using a Gaussian approximation.

Here, the recommended approach in the literature is used to approximate the moments of the Gaussian distribution using the moment generating function $[32,48,49]$. It is used to generate moments of mean value

$$
\mu_{p}=\mu_{A^{\prime} / A} \mu_{\phi^{\prime}}+\sigma_{A^{\prime} / A \phi^{\prime}}^{2}
$$

and variance

$$
\begin{aligned}
& \sigma_{p}^{2}=\mu_{A^{\prime} / A}^{2} \sigma_{\phi^{\prime}}^{2}+\mu_{\phi^{\prime}}^{2} \sigma_{A^{\prime} / A}^{2}+\sigma_{A^{\prime} / A}^{2} \sigma_{\phi^{\prime}}^{2} \\
& +2 \sigma_{A^{\prime} / A \phi^{\prime}}^{2} \mu_{A^{\prime} / A} \mu_{\phi^{\prime}}+\sigma_{A^{\prime} / A \phi^{\prime}}^{2} \sigma_{A^{\prime} / A} \sigma_{\phi^{\prime}},
\end{aligned}
$$

which are used to replace the mean value and variance in the Gaussian approximation. The approximation of the distribution function of $\phi^{\prime} A^{\prime} / A$ is then given by

$$
g_{p}\left(\phi^{\prime} A^{\prime} / A\right)=\frac{1}{\sqrt{2 \pi \sigma_{p}^{2}}} \exp \left(-\frac{\left(\phi^{\prime} A^{\prime} / A-\mu_{p}\right)^{2}}{2 \sigma_{p}^{2}}\right) .
$$

The distribution function of $\chi$ can then be approximated using again preservation of area

$$
h\left(\chi\left(\mu_{p}\right)\right)=\frac{3}{4} \frac{\Omega}{\chi^{2}} \frac{1}{\sqrt{2 \pi \sigma_{p}^{2}}} \exp \left(-\frac{\left(\frac{3 \Omega}{4 \chi}-\mu_{p}\right)^{2}}{2 \sigma_{p}^{2}}\right) .
$$

This distribution is not further studied here, because it is only an approximation of the real distribution. In Section 4, it is more extensively studied comparing it also to the distribution of (4) calculated using a Monte Carlo simulation.

It is important to stress here again, that unlike the distribution based on $\left(\phi^{\prime}\right)^{2}$ and $\left(A^{\prime} / A\right)^{2}$ that $(27)$ is not always a good approximation of the true distribution of $\chi$ based on the product of normal variables $\phi^{\prime}$ and $A^{\prime} / A$.

The approximation can also be extended to include the cylindrical geometry and density. If $\rho$ and $n^{\prime} / n$ are assumed to be deterministic by replacing $\mu_{A^{\prime} / A}=$ $A^{\prime} / A+1 /(2 \rho)+n^{\prime} / n$. On the other hand, if they are assumed also to be random variables a Taylor expansion can be used to include them given that the linearization is a good approximation. However, in this paper only slab-geometry will be considered.

The CDF of (27) is not presented here as it does not have a closed-form solution. However, the CDF can be found by numerically approximating a single integral such that the confidence bounds can still be calculated, which is described in Appendix B.

In the next section, $\chi$ is estimated based on real measurements.

\section{Estimating means and (co-)variances from measurements}

In the next section, we discus how to estimate the diffusion coefficient from real measurements. Therefore, in this section realistic values for the Fourier coefficients and its corresponding variances are calculated. These are acquired from ASDEX 
Upgrade discharge 17175, where the modulated ECRH is deposited off-axis at toroidal normalized radius $\rho_{t}=0.6$. A detailed description of this discharge can be found in $[50,51]$. Here, the distribution function is investigated based on the measurement data and it is explained how to calculate the mean values and variances of the Fourier coefficients. In addition, the mean values and (co-)variances are calculated at two specific spatial locations.

\subsection{Noise distribution of ASDEX Upgrade measurement}

In Section 2.2, it is explained why ECE-measurements are Gaussian distributed. It is possible to verify this using the measurement data from ASDEX Upgrade. Although the time interval where the periodic perturbations are present can be used to extract the variances (see next section), it is unsuitable to determine the PDF due to the low number of periods available. Therefore, the time domain noise is extracted from a time interval without perturbations, which will give the natural noise distribution. The normalized histograms of the ECE-signals considered are presented in Fig. 5 (left). A Gaussian distribution can be recognized, albeit disturbed due to quantization (discretization). However, we are interested in frequency domain properties at the perturbed harmonics $\Omega_{k}$. Therefore, extra random samples are generated, which have the same normalized distribution function as the quantized noise distribution shown in Fig. 5. Then, the Fourier transform is calculated per period, which results in many Fourier coefficients for the ground frequency $\Omega_{1}$. This process is performed for two ECE-measurements at different radial locations. The resulting distributions of these Fourier coefficients are presented in Fig. 5. Both, the real and imaginary parts are Gaussian distributed with very similar variances such that it can be concluded that they obey a CCND. In the case of perturbative measurements other disturbances can also occur, but it is still likely that the underlying distribution is CCND.

\subsection{Estimating the Fourier coefficients and variances}

Here, it is explained how to estimate the Fourier coefficients and variances based on periodic perturbations. The estimated Fourier coefficients are denoted by $\hat{\Theta}\left(\Omega_{k}\right)$ (hat denotes estimates), which can also be seen as the mean value of the Fourier coefficient. There are two possibilities to calculate the mean values of the Fourier coefficients: 1) the Fourier transform can be applied over the entire time interval or 2) the Fourier coefficients can be calculated per period, which are then averaged to find the mean values of the Fourier coefficients $\hat{\Theta}\left(\Omega_{k}\right)=\hat{\mu}_{\Re}\left(\Omega_{k}\right)+i \cdot \hat{\mu}_{\Im}\left(\Omega_{k}\right)$, i.e.

$$
\hat{\Theta}\left(\Omega_{k}\right)=\frac{1}{P} \sum_{p=1}^{P} \Theta^{[p]}\left(\Omega_{k}\right),
$$

where $\Theta^{[p]}\left(\Omega_{k}\right)$ is the Fourier coefficient of the individual period $p$ for frequency $\Omega_{k}$ and $P$ is the total number of periods. Calculating the Fourier transform (FFT) of one period (here $68 \mathrm{~ms}$ ) and averaging over all periods (here $P=10$ ) is equivalent to calculating the Fourier transform of the entire time trace (here $680 \mathrm{~ms}$ ) for the common frequencies (if the number of periods $P$ is integer and without using a window).

The advantage of determining the Fourier coefficients per period $\Theta^{[p]}\left(\Omega_{k}\right)$ is that they can also be used to directly estimate the variances

$$
\hat{\sigma}_{\Re}^{2}\left(\Omega_{k}\right)=\frac{1}{P(P-1)} \sum_{p=1}^{P}\left|\Theta_{\Re}^{[p]}\left(\Omega_{k}\right)-\hat{\Theta}_{\Re}\left(\Omega_{k}\right)\right|^{2},
$$



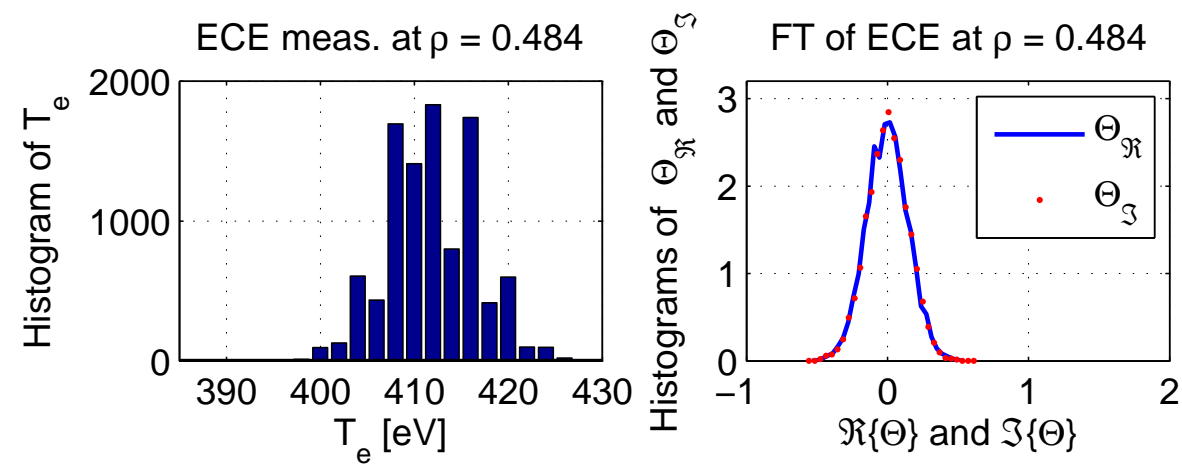

ECE meas. at $\rho=0.473$
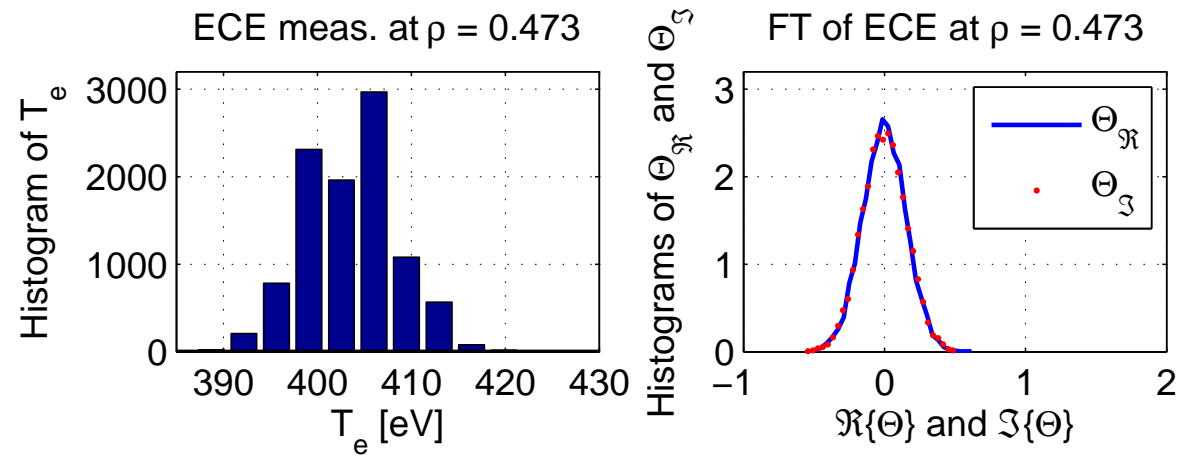

Figure 5. Histograms of AUG 17175 ECE measurements at locations $\rho=0.484$ and $\rho=0.473$ measured between $1.67-2 \mathrm{~s}$ in the plasma and PDFs (left). On the right the histograms of $\Theta_{\Re}$ and $\Theta_{\Im}$ respectively the real and imaginary part of the Fourier coefficients at $14.71 \mathrm{~Hz}$ (first harmonic) respectively at radial locations $\rho=0.484$ and $\rho=0.473$. The right figures are generated using a Monte Carlo based technique. The distributions in time (left figures) are used to generate many samples $(10000$ periods at $14.71 \mathrm{~Hz})$. These periods are Fourier transformed and the real and imaginary parts of the Fourier coefficients at $14.71 \mathrm{~Hz}$ are extracted. These are used to construct the histograms of $\Theta_{\Re}$ and $\Theta_{\Im}$, which clearly show that the resulting distribution is CCND.

where $\Theta_{\Re}^{[p]}$ is the real part of $\Theta^{[p]}$. Similarly, also the variance $\hat{\sigma}_{\Im}^{2}\left(\Omega_{k}\right)$ of the imaginary part can be determined. The covariances $\sigma_{\Re \Im}^{2}\left(\Omega_{k}, \rho_{i}, \rho_{i+1}\right), \sigma_{\Im \Im}^{2}\left(\Omega_{k}, \rho_{i}, \rho_{i+1}\right), \sigma_{\Re \Im}^{2}\left(\Omega_{k}, \rho_{i}\right), \ldots$ can be approximated using [43]

$$
\begin{aligned}
& \hat{\sigma}_{\Im \Re}^{2}\left(\Omega_{k}, \rho_{i+1}, \rho_{i}\right)=\frac{1}{P(P-1)} \sum_{p=1}^{P}\left(\Theta_{\Im}^{[p]}\left(\Omega_{k}, \rho_{i+1}\right)\right. \\
& \left.-\hat{\Theta}_{\Im}\left(\Omega_{k}, \rho_{i+1}\right)\right)\left(\Theta_{\Re}^{[p]}\left(\Omega_{k}, \rho_{i}\right)-\hat{\Theta}_{\Re}\left(\Omega_{k}, \rho_{i}\right)\right) .
\end{aligned}
$$

Here, it is also clear that the variances refer to the variation of the Fourier coefficients with respect to their mean values and the co-variances to the common variations of the Fourier coefficients.

In (16) is chosen to estimate the real and imaginary parts. This is in principle not necessary for the assumption of a CCND as in practice the real and imaginary parts are i.i.d., i.e. $\sigma_{\Re}^{2}\left(\Omega_{k}\right)=\sigma_{\Im}^{2}\left(\Omega_{k}\right)=\hat{\sigma}_{F}\left(\Omega_{k}\right)$ (in practice $\hat{\sigma}_{\Re}^{2}\left(\Omega_{k}\right) \approx \hat{\sigma}_{\Im}^{2}\left(\Omega_{k}\right)$ due to 
uncertainty in the estimates of $\hat{\sigma}_{\Re}^{2}\left(\Omega_{k}\right)$ and $\left.\hat{\sigma}_{\Im}^{2}\left(\Omega_{k}\right)\right)$. However, if the distribution function is different from a CCND, which generally only occurs for distribution functions very different from a Gaussian distribution function with low amount samples in one period, then considering also the imaginary part and real part partly takes this into account. The disadvantage is that the estimates $\sigma_{\Im}^{2}$ and $\sigma_{\Re}^{2}$ will have a higher uncertainty. If one abides by the CCND then it is more common to estimate the variance $\hat{\sigma}_{c}^{2}$ in the complex plane, i.e.

$$
\hat{\sigma}_{c}^{2}\left(\Omega_{k}\right)=\frac{1}{P(P-1)} \sum_{p=1}^{P}\left|\Theta^{[p]}\left(\Omega_{k}\right)-\hat{\Theta}\left(\Omega_{k}\right)\right|^{2} .
$$

where $\hat{\sigma}_{F}^{2}=\hat{\sigma}_{c}^{2}\left(\Omega_{k}\right) / 2$, which follows from (31) by separating the real and imaginary part,

$$
\hat{\sigma}_{c}^{2}\left(\Omega_{k}\right)=\hat{\sigma}_{\Re}^{2}\left(\Omega_{k}\right)+\hat{\sigma}_{\Im}^{2}\left(\Omega_{k}\right),
$$

and using the fact that the real and imaginary part are i.i.d.

Warning: the amplitude and phase per period and its corresponding variances should not be used to derive the overall amplitude and phase. The reason is that $\sigma_{F}^{2}$ is $P$ times larger per period, such that the distributions of amplitude and phase are not necessarily Gaussian. In addition, the phase can also be mapped differently in the complex plane as large error angles are added shifting the phase beyond $-\pi$ or $\pi$, because of the high noise level. This would result in a totally different wrong phase average. Therefore, the amplitude and phase distributions will be given in terms of $\sigma_{\Re}^{2}\left(\Omega_{k}\right)$ and $\sigma_{\Im}^{2}\left(\Omega_{k}\right)$.

\subsection{Resulting $A^{\prime} / A$ and $\phi^{\prime}$ for $A U G 17175$ at $\rho_{t}=0.473$ and $\rho_{t}=0.484$}

Here, based on the previous sections the mean values and variances are calculated based on real measurements from ASDEX-U. These are necessary to calculate $\chi$ with its corresponding confidence. Here, only the procedure to estimate $\chi$ and its confidence bounds is investigated. Therefore, only the confidence at two spatial locations $\rho_{t}=0.473$ and $\rho_{t}=0.484$ for AUG 17175 are investigated. This discharge is chosen as it has many low-frequent harmonics, which thus have more harmonics with acceptable SNRs. On the other hand, the effect of non-diffusive contributions such as convectivity and damping cannot be excluded. The observation that the amplitude and phase do not describe a purely diffusion model for AUG 17175 is also made in [50], in which it is indicated that the heat-pinch exhibited by the amplitude profile is not caused by an actual heat-pinch but is attributed to an effect of the turbulent transport when the temperature profile is just above the heat transport threshold. Therefore, often in the analysis in this paper the mean values are replaced with known values. This not only excludes the effect of these non-diffusive terms, but also helps the interpretation of the results as the true value is known.

First shortly the steps are discussed based on the estimation of the first harmonic $\Omega_{1}$. Based on (28) the Fourier coefficients of the first harmonic can be calculated resulting in $\Theta\left(\rho_{i+1}, \Omega_{1}\right)=1.67+28.75 i$ and $\Theta\left(\rho_{i+1}, \Omega_{1}\right)=2.80+27.42 i$. Then using (15) the mean values $\mu_{A^{\prime} / A}\left(\Omega_{1}\right)=5.75$ and $\mu_{\phi^{\prime}}\left(\Omega_{1}\right)=3.77$ are calculated. The next step is to estimate the covariance matrix using (29) and (30). This results in

$$
\operatorname{cov}\left(\Omega_{1}, \Theta\left(\rho_{i}\right), \Theta\left(\rho_{i+1}\right)\right)=
$$


Table 1. The mean values and variances of $A^{\prime} / A$ and $\phi^{\prime}$ at different $\Omega_{k}$, for time trace $t=3.26-3.94$ and radial locations $\rho_{t}=0.484$ and $\rho_{t}=0.473$.

\begin{tabular}{|c|c||c|c|c|c|c|c|}
\hline & \multicolumn{1}{|c||}{} & \multicolumn{2}{c|}{$A^{\prime} / A$} & \multicolumn{2}{c|}{$\phi^{\prime}$} & $A^{\prime} / A \phi^{\prime}$ & $\chi=5$ \\
\hline$k$ & $\Omega_{k}$ & $\mu_{A^{\prime} / A}\left(\Omega_{k}\right)$ & $\sigma_{A^{\prime} / A}^{2}\left(\Omega_{k}\right)$ & $\mu_{\phi^{\prime}}\left(\Omega_{k}\right)$ & $\sigma_{\phi^{\prime}}^{2}\left(\Omega_{k}\right)$ & $\sigma_{\phi^{\prime} A^{\prime} / A}^{2}\left(\Omega_{k}\right)$ & $\theta^{\prime}\left(\Omega_{k}\right)$ \\
\hline 1 & 92.4 & 5.75 & 0.09 & 3.77 & 0.15 & 0.04 & 3.72 \\
\hline 2 & 184.8 & 6.40 & 0.34 & 4.94 & 0.70 & -0.11 & 5.27 \\
\hline 3 & 277.2 & 8.03 & 1.05 & 5.52 & 1.58 & -0.11 & 6.45 \\
\hline 4 & 369.6 & 12.29 & 3.28 & 7.72 & 3.07 & -0.58 & 7.45 \\
\hline 5 & 462.0 & 12.55 & 7.68 & 5.95 & 13.90 & -0.72 & 8.32 \\
\hline
\end{tabular}

$$
\left[\begin{array}{cccc}
0.49 & -0.09 & 0.45 & -0.09 \\
-0.09 & 0.28 & -0.10 & 0.30 \\
0.45 & -0.10 & 0.43 & -0.09 \\
-0.09 & 0.30 & -0.09 & 0.31
\end{array}\right]
$$

which including the mean values will be used extensively in the next section. The corresponding covariance matrix of $A^{\prime} / A$ and $\phi^{\prime}$ is calculated using (17). This gives

$$
\operatorname{cov}\left(\Omega_{1}, A^{\prime} / A, \phi^{\prime}\right)=\left[\begin{array}{ll}
0.09 & 0.04 \\
0.04 & 0.15
\end{array}\right],
$$

where the diagonal terms are the variance of phase $\sigma_{\phi^{\prime}}^{2}$ and amplitude $\sigma_{A^{\prime} / A}^{2}$. The offdiagonal term is the covariance denoted by $\sigma_{A^{\prime} / A \phi^{\prime}}^{2}$, which is sometimes also expressed in terms of the Pearson factor $\rho_{\text {pearson }}=0.3356$ [31].

The steps to calculate $\mu_{A^{\prime} / A}\left(\Omega_{k}\right), \mu_{\phi^{\prime}}\left(\Omega_{k}\right)$, and $\operatorname{cov}\left(\Omega_{1}, A^{\prime} / A, \phi^{\prime}\right)$ are repeated for the first five harmonics $\Omega_{k}$, which fulfill the necessary SNRs. These are presented in Table 1 and are extensively used when combining harmonics.

If the mean values $\mu_{A^{\prime} / A}\left(\Omega_{1}\right)$ and $\mu_{\phi^{\prime}}\left(\Omega_{1}\right)$ and its corresponding variances $\sigma_{\phi^{\prime}}^{2}$ and $\sigma_{A^{\prime} / A}^{2}$ are compared, then the confidence bounds do not overlap. This also indicates that indeed the measurements cannot be described by a model with diffusivity only. Hence, this uncertainty analysis also offers a zero-order test to see if the measurements fit such a model. As here the performance of the different methods are investigated a known value of $\chi$ is used. The first harmonic has generally the best SNR, hence is a good choice for $\chi$. As the phase is considered less sensitive to calibration errors the phase has been chosen resulting in $\chi=4.88$. This has been rounded to the closest integer, i.e. $\chi=5$, to simplify the interpretation of the different figures and errors. Then, (1) is rewritten to express $\mu_{\phi^{\prime}}(\chi=5)$. This results in new means for $\mu_{\phi^{\prime}}$ denoted as $\theta^{\prime}$. The difference between $\mu_{\phi^{\prime}}\left(\Omega_{k}\right)$ and the new $\theta^{\prime}\left(\Omega_{k}\right)$ are not so large. Therefore, the original variances $\sigma_{k}^{2}$ are retained. These new values are also included in Table 1 and are used to study the estimation of $\chi$ in the next section.

\section{Estimating $\chi$}

In this section a number of aspects of estimating $\chi$ are discussed and $\chi$ is estimated based on real measurements: a) how to combine $A^{\prime} / A$ and $\phi^{\prime}$ optimally in the case of diffusivity only; b) how to combine different harmonics such that the resulting estimate of $\chi$ has a small bias and high accuracy. This is investigated for the the different relationships in Section 2.1. Finally, the necessary steps to estimate $\chi$ are summarized at the end of this section. 


\subsection{Combining amplitude and phase estimates}

Here, the different possibilities proposed to combine phase and amplitude are compared. As the interest goes out to the statistical properties it is assumed that only the diffusivity is present. Therefore, in the analysis the mean values are also replaced to exclude the effect of non-diffusive terms. The main question of this subsection is how to estimate $\chi$ using amplitude and phase, which can be seen as independent measurements of $\chi$ containing correlated noise.

In Section 4.3 it has been shown that the cross-correlation between $A^{\prime} / A$ and $\phi^{\prime}$ is significant. As such this cross-correlation can be exploited to increase the accuracy of the $\chi$ estimate. Therefore, the generalized weighted mean can be used to give a resulting combination of $A^{\prime} / A$ and $\phi^{\prime}$ with minimum variance. The Gauss-Markov theorem states that it results in minimum variance for unbiased estimators of the mean value [52]. This generalized weighted mean is calculated as follows

$$
\mu_{M}=\sigma_{M}^{2} W^{T} \operatorname{cov}\left(A^{\prime} / A, \phi^{\prime}\right)^{-1}\left[\mu_{A^{\prime} / A}\left(\Omega_{1}\right), \mu_{\phi^{\prime}}\left(\Omega_{1}\right)\right]^{T},
$$

and its corresponding variance is determined with

$$
\sigma_{M}^{2}=\left(W^{T} \operatorname{cov}\left(A^{\prime} / A, \phi^{\prime}\right)^{-1} W\right)^{-1} \text { with } W=\left[\begin{array}{ll}
1 & 1
\end{array}\right]^{T} .
$$

There are three different possibilities presented to combine $A^{\prime} / A$ and $\phi^{\prime}$ namely $\chi_{s 3}$ in (2), $\chi_{s 4}$ in (4), and the generalized weighted mean in (35). The product (4) and (5) are theoretically the same for one harmonic. Therefore, only (4) has been presented here. The three different possibilities will be compared using a Monte Carlo simulation which uses (33) and mean Fourier coefficients. In a Monte Carlo simulation samples are generated from a distribution, which can be seen as possible measurements. Then, these samples are used to calculate $\chi$ using the different proposed relationships. This process is repeated many times such that the PDF of the diffusivity estimates can be generated. The analytic distributions for the generalized mean and (27) are also presented.

Three simulations are performed for different cases: a) using the original measurement data; b) a simulation where the original variances are retained, but the mean values are replaced by $\Theta\left(\rho_{i+1}, \Omega_{1}\right)=1.67+28.75 i$ and $\Theta\left(\rho_{i+1}, \Omega_{1}\right)=$ $2.80+27.42 i$ in (4.3) such that both mean values $\mu_{A^{\prime} / A}\left(\Omega_{1}\right)$ and $\mu_{\phi^{\prime}}\left(\Omega_{1}\right)$ will give $\chi=5$ when the variance is zero; c) a simulation where next to the replaced mean values also the second diagonal element of $\operatorname{cov}\left(\Omega_{1}, \Theta\left(\rho_{i}\right), \Theta\left(\rho_{i+1}\right)\right)$ is replaced by the first diagonal element, i.e. $\sigma_{\Im}^{2}\left(\rho_{i}\right)=0.4904$ in $(33)$.

Fig. 6 shows the resulting PDFs of the diffusivity denoted by $h(\chi)$. These PDFs take the cross-correlation between phase and amplitude into account naturally, because it is generated based on samples (except $\mu_{M}$ ). The confidence bounds corresponding to the distributions presented in Fig. 6 are given in Table 2. Interestingly, in Fig. 6(b) the PDFs are almost the same, the reason is that their mean values have been fixed to be the same and the covariance matrix results in an almost equal variance for both amplitude and phase (after taking cross-correlation into account). This means that there is hardly any difference between the different relationships to calculate $\chi$. Although, if considered carefully it is clear that $h\left(\chi\left(\mu_{M}\right)\right)$ has the smallest confidence interval, which can also be concluded from Table 2 . Also, it is clear that $h\left(\chi\left(\mu_{p}\right)\right)$ is an approximation as it clearly deviates from the distribution based on (4).

The other two cases give more interesting results. The sum of $A^{\prime} / A$ and $\phi^{\prime}$ gives again a Gaussian distribution, which has smaller uncertainty compared to the product 
(a)
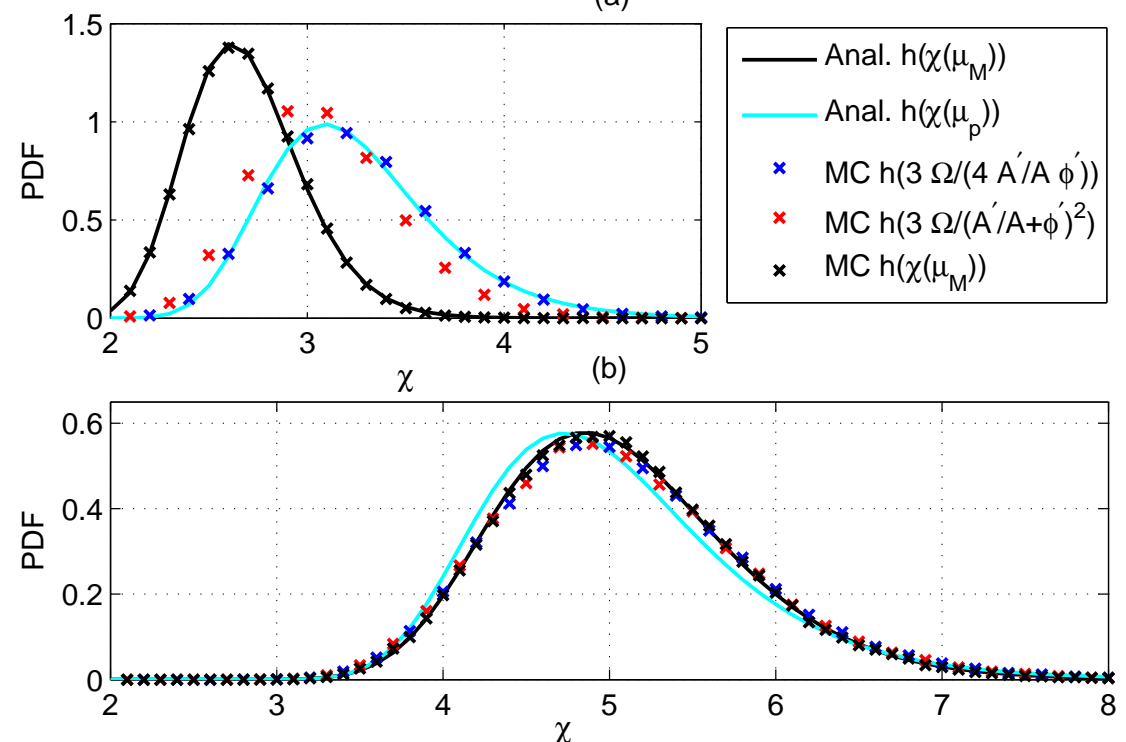

(c)

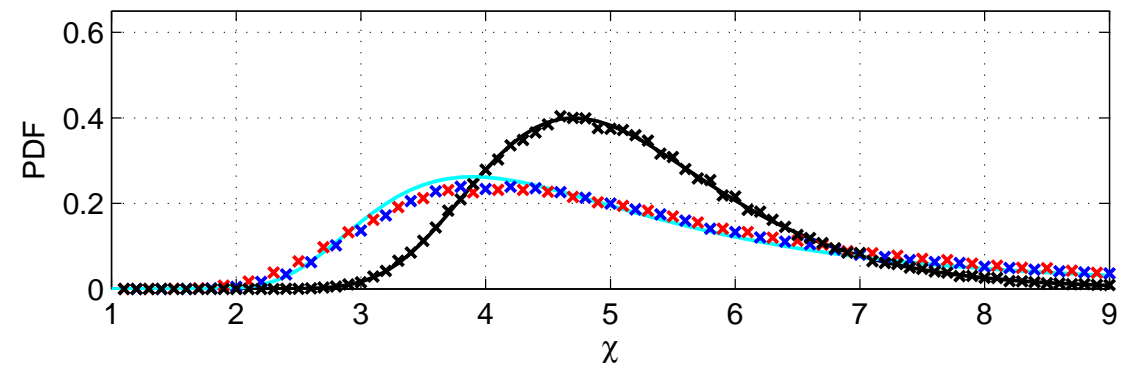

Figure 6. Normalized histograms (PDFs) for the four proposed calculation methods to combine phase and amplitude, i.e. (4) represented using (blue) $\times$, (3) represented using using (red) $\times$, and (37) using (black) $\times$. The distributions are generated using Monte Carlo simulations (MC). Also the analytic approximations of the distribution functions $h\left(\chi\left(\mu_{M}\right)\right)$ and $h\left(\chi\left(\mu_{p}\right)\right)$ are presented using a black and cyan line respectively. These distributions are solely based on analytical calculations using (37) to find $\mu_{M}$ with corresponding $\sigma_{M}^{2}$ and (20) to calculate the PDF of $\chi$, i.e. $h\left(\chi\left(\mu_{M}\right)\right)$. In the product case $(24)$ and $(25)$ are used to calculate $\mu_{p}$ and $\sigma_{p}^{2}$, which are used in (27) to calculate $h\left(\chi\left(\mu_{p}\right)\right)$. Three simulations are presented: (a) using the original mean value and variance; (b) using the corrected mean values, but the original covariance matrix; (c) using the corrected mean value and a different covariance matrix. 
Table 2. The numerically determined central interval confidence bounds for the diffusivity $\chi_{M C}$ based on a Monte Carlo simulations (MC) for the different methods to combine phase and amplitude. In addition, the analytically calculated confidence bounds for the generalized weighted mean method $\chi_{\text {anal }}\left(\mu_{M}, \sigma_{M}^{2}\right)$ and the product approximation $\chi_{a n a l}\left(\mu_{p}, \sigma_{p}^{2}\right)$ are presented. The results are presented for the measurements (left) and the results using the adjusted Fourier coefficients and adjusted covariance matrix (right). Note, that the $\chi$ value is calculated using the corresponding equations and are not based on the Monte Carlo analysis.

\begin{tabular}{|c|c|c|c|c|c|c|}
\hline & \multicolumn{3}{|c|}{ Fig. 6(a) } & \multicolumn{3}{c|}{ Fig. 6(c) } \\
\hline conf. bnd & $2.5 \%$ & $\chi$ & $97.5 \%$ & $2.5 \%$ & $\chi$ & $97.5 \%$ \\
\hline$\chi_{M C}=\frac{3 \Omega}{4 \phi^{\prime} A^{\prime} / A}$ & 2.50 & 3.20 & 4.19 & 2.66 & 5.00 & 17.82 \\
\hline$\chi_{\text {anal }}\left(\mu_{p}, \sigma_{p}^{2}\right)$ & 2.54 & 3.19 & 4.29 & 2.67 & 4.89 & 19.20 \\
\hline$\chi_{M C}=\frac{3 \Omega}{\left(\phi^{\prime}+A^{\prime} / A\right)^{2}}$ & 2.43 & 3.06 & 3.89 & 2.57 & 5.00 & 14.12 \\
\hline$\chi_{M C}=\frac{3 \Omega}{4 \mu_{M}^{2}}$ & 2.16 & 2.68 & 3.30 & 3.43 & 5.00 & 7.84 \\
\hline$\chi_{\text {anal }}\left(\mu_{M}, \sigma_{M}^{2}\right)$ & 2.17 & 2.68 & 3.33 & 3.44 & 5.00 & 7.88 \\
\hline
\end{tabular}

of two Gaussian distributed variables for the relevant variance ranges. The best result is achieved using (35) it has the smallest variance (uncertainty). This is especially clear in Fig. 6(c). Moreover, this distribution can also be analytically calculated using the variance (36). This is also supported by the confidence bounds in Table 2 .

Summarizing, the generalized weighted mean gives the best result, which is consistent with theoretical predictions.

\subsection{Combining different harmonics $\phi^{\prime}$ and $A^{\prime} / A$ only}

In this section, methods are discussed to combine different harmonics. They are presented on the basis of the phase derivative distribution only, because the replaced means $\theta^{\prime}\left(\Omega_{k}\right)$ are used to exclude the effect of non-diffusive terms. The same conclusions can also be drawn if the amplitude information is used. The calculations are based on the values presented in Table 1.

In this Monte Carlo simulation from the five Gaussian distributions with mean values $\mu_{\phi^{\prime}}\left(\Omega_{k}\right)$ and variances $\sigma_{\phi^{\prime}}^{2}\left(\Omega_{k}\right)$ are used based on 10000 samples. The simplest method of combining different harmonics is by averaging the diffusivity estimates calculated for every harmonic, i.e. $E\{\chi\}$. The resulting PDF of $\chi$ is then denoted by $h(E\{\chi\})$ and it is presented in Fig. 7 with (blue) crosses. The resulting PDF of $\chi$ is non-symmetric and has a long tail. The reason is that $h(E\{\chi\})$ is the result of averaging five PDFs of $\chi\left(\Omega_{k}\right)$, which are distributed according to (20). These individual PDFs of $\chi\left(\Omega_{k}\right)$ already have a long tail and contain a bias. This bias depends on the individual variances $\sigma_{\phi^{\prime}}^{2}\left(\Omega_{k}\right)$. This tail and bias are the result of taking the squared reciprocal of the phase derivative and are retained when averaging these estimates.

A better option is to directly average the phase derivatives, because their distributions are still unbiased and Gaussian. However, the diffusivity does not only depend on $\phi^{\prime}$, but also on the frequency $\Omega$ in (1). Therefore, the Gaussian distributions can only be averaged if they are first weighted with the frequencies, i.e. $\mu_{\phi^{\prime}}\left(\Omega_{k}\right) / \sqrt{\Omega_{k}}$ and $\sigma_{\phi^{\prime}}^{2}\left(\Omega_{k}\right) / \Omega_{k}$. Then, a new Gaussian distribution is found and it is used to calculate the resulting PDF of $\chi$, which is denoted by $h\left(\chi\left(E\left\{\mu_{\phi^{\prime}}\left(\Omega_{k}\right) / \sqrt{\Omega_{k}}\right\}\right)\right)$. 


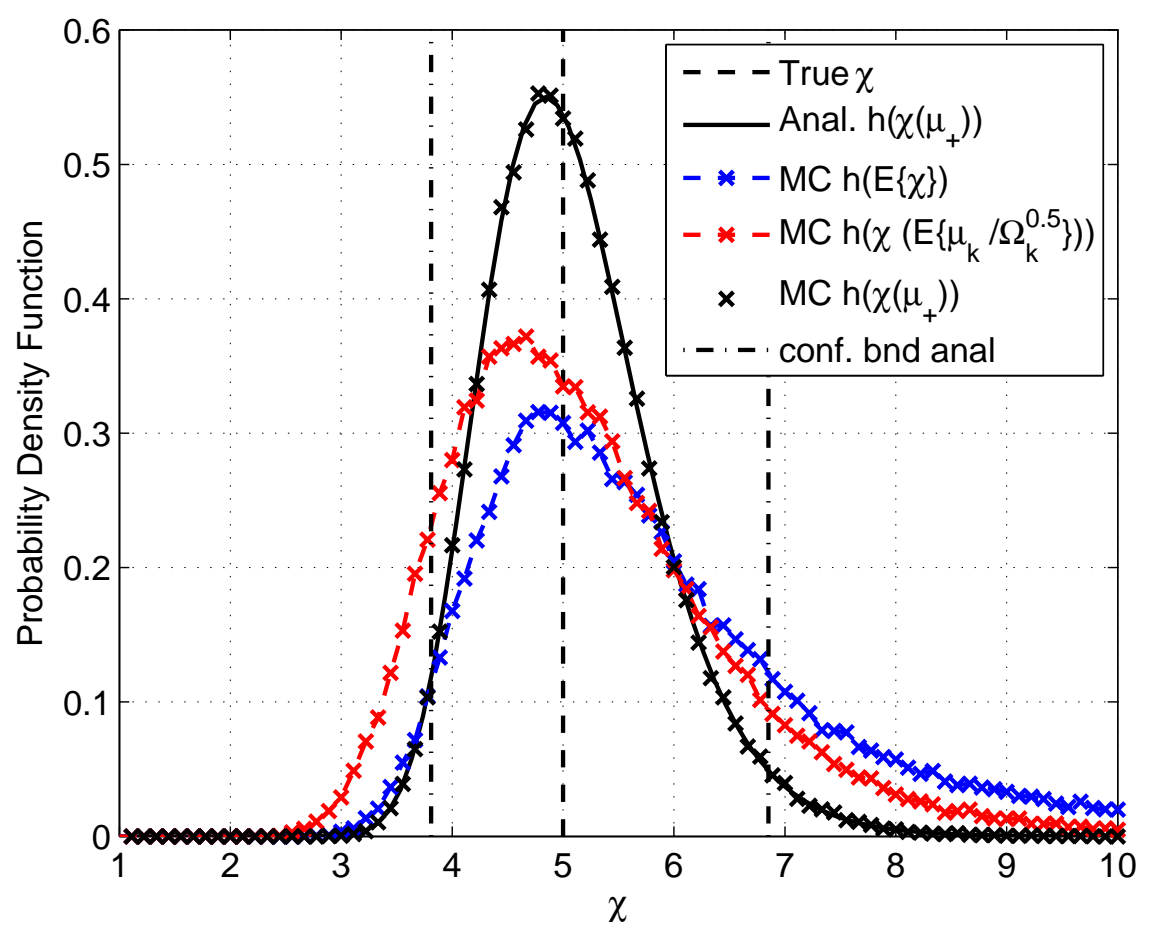

Figure 7. Normalized histograms (PDFs) for the three proposed calculation methods to calculate $\chi$ from either phase (or amplitude) using multiple harmonics generated using a Monte Carlo simulation (MC). The PDF $h(E\{\chi\})$ represented by $x$ is calculated using the mean value of the different $\chi$ 's of the different harmonics. The PDF $h\left(\chi\left(E\left\{\mu_{k} / \sqrt{\Omega_{k}}\right\}\right)\right)$ represented by $\times$ gives the PDF of $\chi$ using the weighted average of the phase derivatives using only $\sqrt{\Omega_{k}}$. The PDF $h\left(\chi\left(\mu_{+}\right)\right)$is represented by $\times$, where a weighted average in (37) is used to calculate $\chi$. In addition, the theoretically determined PDF $h\left(\chi\left(E\left\{\mu_{+}\right\}, E\left\{\sigma_{+}^{2}\right\}\right)\right)$ based on the mean value of $\mu_{+}$and the mean value of $\sigma_{+}^{2}$ is presented. The true value of $\chi$ is presented (dashed) and the $95 \%$ confidence interval for $h\left(\chi\left(\mu_{+}\right)\right.$) is presented by the dashed-dotted vertical lines (left $2.5 \%$ and right $97.5 \%)$, which are calculated analytically.

In this case, the resulting distribution can be determined analytically by calculating the new mean value and variance of the combined Gaussian distribution and then using (20) again. Here, we have only used the Monte Carlo simulation to determine the PDF, which is shown in Fig. 7 using $\times$.

It is clear that the side tail is still present, but has become smaller. The diffusivity has become more certain due to the smaller side tail, but the uncertainty region has shifted to the left. However, this method does not take the uncertainty on $\phi^{\prime}$ into account. This means that there is no difference between estimates of the phase derivative with small variance compared to phase derivatives with high variance. In other words, the uncertain higher harmonics contaminate the certain low-harmonics in the diffusivity estimates.

The diffusivity estimate can be improved by taking the variance of the different harmonics into account, which have been estimated from the periodic measurements. 
Table 3. The central interval confidence bounds for $\chi_{M C}$ using the different combination methods based on a Monte Carlo simulations (MC). In addition, the analytically calculated confidence bounds for the weighted mean method $\chi_{\text {anal }}$.

\begin{tabular}{|c|c|c|c|}
\hline conf. bnd & $2.5 \%$ & $\chi$ & $97.5 \%$ \\
\hline$\chi_{M C}(E\{\chi\})$ & 3.73 & 6.37 & 45.09 \\
\hline$\chi_{M C}\left(E\left\{\mu_{k} / \sqrt{\Omega_{k}}\right\}\right)$ & 3.32 & 6.02 & 8.32 \\
\hline$\chi_{M C}\left(\mu_{\dagger}\right)$ & 3.81 & 5.11 & 6.84 \\
\hline$\chi_{\text {anal }}\left(E\left\{\mu_{+}\right\}, E\left\{\sigma_{+}^{2}\right\}\right)$ & 3.81 & 5.11 & 6.84 \\
\hline
\end{tabular}

This results in the following weighted mean with $\mu_{k}=\mu\left(\Omega_{k}\right)$, which is also applicable for the amplitude

$$
\mu_{+}=\frac{\sum_{k=1}^{K} w_{k} \mu_{k} \Omega_{k}^{-0.5}}{\sum_{k=1}^{K} w_{k}} \text { with } w_{k}=\frac{\Omega_{k}}{\sigma_{k}^{2}} .
$$

This type of weighting gives the Maximum Likelihood Estimate of the mean value for Gaussian distributed variables [31], which has the smallest variance when combining a number of independent Gaussian distributions. Note, that if the cross-correlation between harmonics is zero, as is the case, the generalized weighted mean in (35) reduces to (37). In Fig. 7 the resulting PDF of $\chi$ using the weighted average, $h\left(\chi\left(\mu_{\dagger}\right)\right)$ (black $\times$ ). It is clear that the tail has been reduced significantly such that the diffusivity estimate has become much more certain. The use of the weighted mean gives the best result and should be used to combine the different harmonics in case of a purely diffusive model.

The distribution $h\left(\chi\left(\mu_{+}\right)\right)$can also be determined analytically by calculating the variance $\sigma_{\dagger}^{2}$

$$
\sigma_{+}^{2}=\frac{1}{K-1} \frac{\sum_{k=1}^{K} w_{k}\left(\mu_{k} \Omega_{k}^{-0.5}-\mu_{+}\right)^{2}}{\sum_{k=1}^{K} w_{k}},
$$

taking into account that $\sigma_{+}^{2}$ is an estimate [31]. The confidence bounds are calculated by setting the mean value $\mu_{\gamma}=\mu_{+}$, the variance $\sigma_{\gamma}^{2}=\sigma_{+}^{2}$, and $\Omega=1$ in (23). They can also be used to calculate the analytic PDF, which is plotted in Fig. 7. The analytic PDF (solid black line) is the same as the PDF constructed using the Monte Carlo simulations.

A comparison of the different combination methods can also be made using the confidence bounds, which are numerically determined from the Monte Carlo simulations. These confidence bounds are summarized in Table 3. It again shows that the weighted mean gives the best result and that in this case the accuracy of the diffusivity estimate is increased 13 times compared to averaging the diffusivity estimate. Note that, the diffusivity estimate still has a tailed distribution, which is caused by the method of calculation. It is possible to avoid this tail, but this would require a different method to determine $\chi$. Such methods using an implicit scheme exist [31, 43], but are out of scope for this paper.

For the experimental data using both amplitude and phase (no damping), the resulting mean value and variance are $\mu_{\dagger}=0.48$ and $\sigma_{\dagger}^{2}=5.93 \cdot 10^{-4}$ based on the first five frequencies. This is smaller than any of the individual (frequency weighted) variances. The corresponding diffusivity is $\chi=3.29$ with the $95 \%$ confidence bounds $\chi_{\min }=2.72$ and $\chi_{\max }=4.06$. The confidence bounds are larger than that of the first 
harmonic in absolute sense. This is a consequence of the non-linear dependence of the confidence bounds on the mean value.

\subsection{Combining different harmonics using the product $\phi^{\prime} A^{\prime} / A$}

In section 5.2, we have established that for purely diffusive models the combination of the generalized weighted mean to combine $A^{\prime} / A$ and $\phi^{\prime}$ and weighted mean to combine the harmonics results in the $\chi$ estimate with the smallest variance for the compared methods. However, in case no longer a purely diffusive model is assumed, but also damping needs to be included then only (4) and (5) can be used in a slab geometry. Therefore, here it is investigated how to reduce the uncertainty using (4) and (5) to estimate $\chi$.

The product $A^{\prime} / A \phi^{\prime}$ is used to exclude the effect of damping as such the amplitude and phase can no longer be used to reduce the uncertainty using the generalized mean. In principle, one harmonic would suffice to estimate the damping, which then could be used in the weighting process. However, such a weighting is difficult to apply in direct calculations of $\chi$. Instead, the product will be applied for every harmonic. This means that for five harmonics, four degrees of freedom are ignored, which in principle could have been used to reduce the uncertainty. Here, is chosen to disregard this loss in optimality and try to combine the different harmonics based on (4) and (5). Therefore, the values from Table 1 are used again using the original measurement values. Again, three possibilities are compared using a Monte Carlo simulation based $\mu_{A^{\prime} / A}\left(\Omega_{k}\right)$, $\mu_{\phi^{\prime}}\left(\Omega_{k}\right)$, and $\left.\operatorname{cov}\left(\Omega_{k}, A^{\prime} / A, \phi^{\prime}\right): 1\right)$ the simple average of the $\chi$ estimates per harmonic using (4); 2) the weighted average of $A^{\prime} / A \phi^{\prime}$ taking only $\Omega_{k}$ into account; 3 ) the weighted average using the Gaussian approximations with mean (24) and variance (25) taking both $\Omega_{k}$ and $\operatorname{cov}\left(\Omega_{k}, A^{\prime} / A, \phi^{\prime}\right)$ into account. The product of $A^{\prime} / A, \phi$ is used, thus a weighting with $\Omega_{k}$ is necessary instead of $\sqrt{\Omega_{k}}$ such that (37) and (38) become for the weighted mean of the product

$$
\mu_{m p}=\frac{\sum_{k=1}^{K} w_{k} \mu_{p}\left(\Omega_{k}\right) / \Omega_{k}}{\sum_{k=1}^{K} w_{k}} \text { with } w_{k}=\frac{\Omega_{k}^{2}}{\sigma_{k}^{2}},
$$

and

$$
\sigma_{m p}^{2}=\frac{1}{K-1} \frac{\sum_{k=1}^{K} w_{k}\left(\mu_{p}\left(\Omega_{k}\right) / \Omega_{k}-\mu_{m p}\right)^{2}}{\sum_{k=1}^{K} w_{k}} .
$$

In the Monte Carlo analysis 100000 times five mean values are generated and these three techniques are applied to analyze the difference again. In addition, the analytic Gaussian approximation is calculated by substituting $\mu_{m p}$ and $\sigma_{m p}$ into $(27)(\Omega=1)$. The results are presented in Fig. 8. The corresponding confidence bounds are presented in Table 4 The results are similar to that in Section 5.2. Taking simply the average results in a long tail distribution function. This also implies that the use of (5) should be avoided if more harmonics are combined. Another interesting aspect is that the lower bound is negative. Of course this is physically not possible, but it is a result of the fact that the product $A^{\prime} / A \phi^{\prime}$ can become negative.

Avoiding the averaging long tail distributions by averaging first $\mu_{\phi^{\prime}}\left(\Omega_{k}\right) \mu_{A^{\prime} / A}\left(\Omega_{k}\right)$ using weight $\Omega_{k}$ increases the confidence on the estimate. This is further increased when using the weighting based on the Gaussian approximations. Here, $\mu_{p}$ is used in the Monte Carlo analysis instead of simply the product $\left(\sigma_{A^{\prime} / A \phi^{\prime}}^{2}\right.$ difference). The reason is that this compares to the Gaussian approximation method used in the analytic 


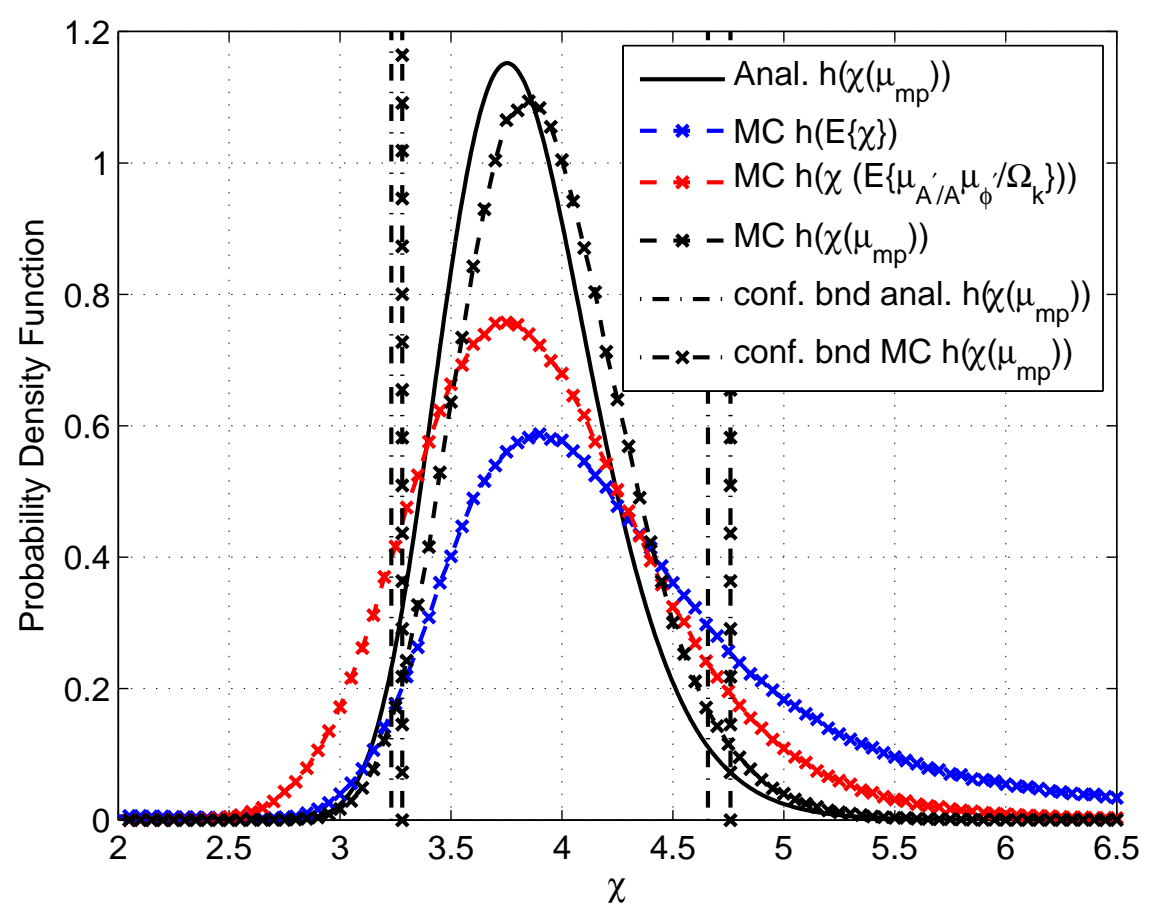

Figure 8. Normalized histograms (PDFs) for the three proposed calculation methods to calculate $\chi$ from either phase (or amplitude) using multiple harmonics generated using a Monte Carlo simulation (MC). The PDF $h(E\{\chi\})$ represented by $\times$ is calculated using the mean value of the different $\chi^{\prime}$ 's of the different harmonics. The PDF $h\left(\chi\left(E\left\{\mu_{\phi^{\prime}}\left(\Omega_{k}\right) \mu_{A^{\prime} / A}\left(\Omega_{k}\right) / \Omega_{k}\right\}\right)\right)$ represented by $\times$ gives the PDF of $\chi$ using the weighted average using only $\Omega_{k}$. The PDF (MC) $h\left(\chi\left(\mu_{m p}\right)\right)$ is represented by $\times$, where a weighted average in (39) is used to calculate $\chi$. In addition, the theoretically determined PDF $h\left(\chi\left(\mu_{m p}\right)\right)$ based on the mean value of $\mu_{m p}$ and the mean value of $\sigma_{m p}^{2}$ is presented. The $95 \%$ confidence intervals (left $2.5 \%$ and right $97.5 \%$ ) are presented using dasheddotted lines for the analytical approximation based on $h\left(\chi\left(\mu_{m p}, \sigma_{m p}^{2}\right)\right)$ and are presented using dashed-dotted lines with $\times$ for $(\mathrm{MC}) h\left(\chi\left(\mu_{m p}\right)\right)$. The latter is based on numerical calculations.

Table 4. The central interval confidence bounds belonging to the lines in Fig. 8 . The bounds based on Monte Carlo simulations $\chi_{M C}$ are calculated numerically. In addition, the analytically calculated confidence bounds for the weighted mean method $\chi_{\text {anal }}\left(\mu_{m p}, \sigma_{m p}\right)$ are presented.

\begin{tabular}{|c|c|c|c|}
\hline conf. bnd & $2.5 \%$ & $\chi$ & $97.5 \%$ \\
\hline$\chi_{M C}(E\{\chi\})$ & -2.07 & 3.97 & 11.26 \\
\hline$\chi_{M C}\left(E\left\{\mu_{\phi^{\prime}} \mu_{A^{\prime} / A} / \Omega_{k}\right\}\right)$ & 3.97 & 3.81 & 5.23 \\
\hline$\chi_{M C}\left(\mu_{m p}\right)$ & 3.28 & 3.82 & 4.76 \\
\hline$\chi_{\text {anal }}\left(\mu_{m p}, \sigma_{m p}\right)$ & 3.23 & 3.82 & 4.66 \\
\hline
\end{tabular}


calculation of $\chi$ using the product. The analytic calculation clearly different from the one generated by the Monte Carlo analysis due to the Gaussian approximations used. If the confidence bounds are considered then it is clear that the under bound is over approximated and the upper bound is under approximated. The reason is that the product of $A^{\prime} / A \phi^{\prime}$ is skewed with a long tail to the right, which is not captured by the Gaussian approximation. However, it is also clear that the difference between the confidence bounds of the analytic calculation and the Monte Carlo analysis is small. This means that this method can be used to estimate $\chi$ and its confidence bounds. However, if the skewness of $A^{\prime} / A \phi^{\prime}$ is large it starts to deviate from the true confidence bounds.

In this paper is chosen to use the moment generating function (Section 3.3) instead of a Taylor expansion (Section 2.5) to approximate the mean value and the variance for the product $A^{\prime} / A \phi^{\prime}$. The advantage of this approach over the Taylor expansion approach is that the confidence bounds found are smaller than that of the Taylor expansion both numerically for the Monte Carlo analysis and analytically. In addition, the distribution function, thus also the confidence bounds, are better approximated using (27) when different harmonics are combined using the moment generating function. On the other hand, in (24) an error on the mean is introduced

(bias term: $\sigma_{A^{\prime} / A \phi^{\prime}}^{2}$ ). So for a better estimate of the mean value it is sometimes desirable to use the Taylor expansion instead.

\subsection{Calibration errors}

Measurements can also be prone to calibration errors. These errors cannot be quantified by studying the perturbative measurements. They are constant for the entire time trace, otherwise the fluctuation would increase the variance. However, they will influence the uncertainty of the parameter estimation.

The amplitude errors can be modeled by introducing scaling factors $\alpha$ and $\beta$, that describe the calibration error in the measured amplitude $\hat{A}$, such that $\hat{A}_{1}=\alpha A_{1}$ and $\hat{A}_{2}=\beta A_{2}$. Note, that the calibration error influences only the gain and as such is constant for all frequencies. Hence, by applying a weighted average similar to (37) for the amplitude measurements an overall $\hat{A}_{1}$ or $\hat{A}_{2}$ can be determined. The new amplitude with calibration error can then be substituted in (2) giving

$$
\chi=\frac{3}{4} \Omega\left(\ln \left(\alpha \hat{A}_{2} / \beta \hat{A}_{1}\right) / \Delta \rho\right)^{-2},
$$

and rewriting yields

$$
\chi=\frac{3 \Omega}{4 \Delta \rho^{2}}\left(\ln (\alpha / \beta)+\ln \left(\hat{A}_{2} / \hat{A}_{1}\right)\right)^{-2} .
$$

The resulting uncertainty on $\chi$ depends on what $\alpha$ and $\beta$ exactly represent. If $\alpha$ and $\beta$ are stochastic, in the sense that they can be presented by a distribution independent of time, and $\ln (\alpha / \beta)$ can be approximated by a Gaussian the extension is straightforward. However, if $\ln (\alpha / \beta)$ cannot be approximated well by a Gaussian, the calculation becomes more complicated and probably numerical tools are necessary e.g. Monte Carlo analysis. Note, that in practice it can be even more cumbersome due to possible correlations between $\alpha$ and $\beta$. On the other hand, if the calibration errors $\alpha$ and $\beta$ are the same for both radial locations the calibration error vanishes.

Commonly, it is presumed that the phase is insensitive to calibration errors. Although, this is true for the mean value, it is not true if $\phi$ is treated as being 


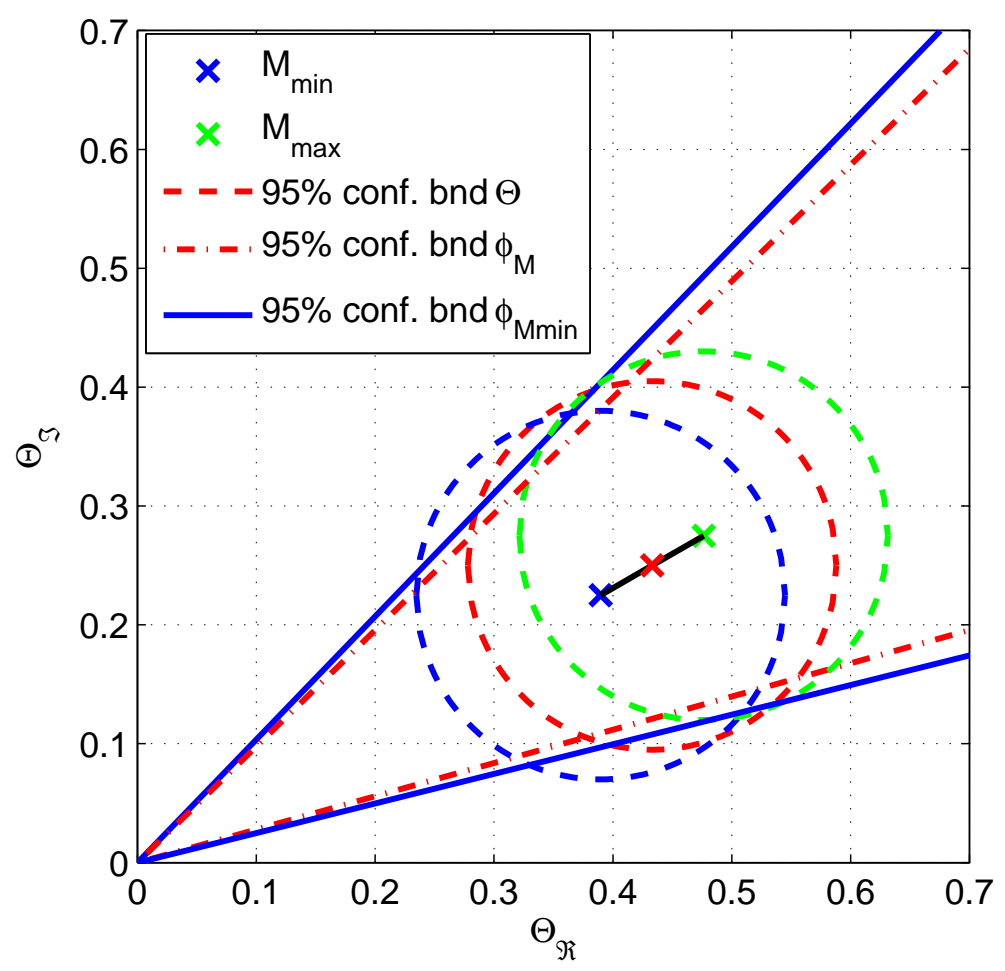

Figure 9. Circular confidence bounds for $M=0.5$ and $\theta=\pi / 6$, and the new circular confidence bounds for amplitude errors of $10 \%$, i.e. $M_{\min }=0.45$ (blue) and $M_{\max }=0.55$ (green). In addition, the phase confidence bounds are presented for $M$ and $M_{\min }$ only, which clearly shows that if $M$ is smaller due to a calibration error, i.e. $M_{\min }$, the uncertainty on the phase increases, which is expressed through the broader confidence bounds.

stochastic. The reason is that the variance of the phase depends on the amplitude (14), which is a direct consequence of the fact that amplitude and phase are correlated quantities. This can be understood if one considers Fig. 1 again, but with varying amplitude $M$ as a result of calibration errors. Fig. 9 clearly shows that in case of a calibration error also the uncertainty on the phase is different. The difference depends on the mean value of the amplitude and the variance. However, considering uncertainty on the variance is rather cumbersome. Therefore, in practice, it is easier to replace the mean value $M$ by the minimal possible $M_{\min }$ due to calibration errors, which gives a conservative confidence bound on $\phi$.

The effect of calibration errors is of a very different nature than the issues described in this paper. Therefore, it will not be included in the analysis of the measurement data.

\subsection{Summary estimating $\chi$ with confidence}

Here, a summary is given of the necessary steps to calculate the overall diffusion coefficients: 
(i) First, calculate the mean value and covariance matrices of the different excited frequencies $\Omega_{k}$.

(ii) Calculate the phase and amplitudes and determine if they can be approximated by Gaussian distributions ( $\mathrm{SNR}>5$ ).

(iii) Determine the mean values $\mu_{A^{\prime} / A^{\prime}}\left(\Omega_{k}\right)$ and $\mu_{\phi^{\prime}}\left(\Omega_{k}\right)$ and the covariance matrix $\operatorname{cov}\left(A^{\prime} / A, \phi^{\prime}\right)$ for every harmonic using the Jacobian, which is described in Section 2.5.

(iv) Make a distinction between $(\mathrm{a})\left(\phi^{\prime}\right)^{2}$ and $\left(A^{\prime} / A\right)^{2}$ and (b) the product of $\phi^{\prime} A^{\prime} / A$ (including cylindrical approximation).

(a) Case: $\left(\phi^{\prime}\right)^{2}$ and $\left(A^{\prime} / A\right)^{2}$

1. Calculate the generalized weighted mean for every harmonic using (35) and its variance using (36). If phase and amplitude are analyzed separately skip this step.

2. Combine the different mean values $\mu_{M}\left(\Omega_{k}\right)$ and variances $\sigma_{M}^{2}\left(\Omega_{k}\right)$ using (37) to determine $\mu_{+}$and its variance $\sigma_{+}^{2}$ using (38).

3. Calculate the overall diffusivity

$$
\chi=\frac{3}{4} \mu_{+}^{-2} .
$$

4. The corresponding confidence bounds are calculated by replacing $\mu_{\gamma}=$ $\mu_{+}, \sigma_{\gamma}^{2}=\sigma_{+}^{2}$, and $\Omega=1$ in (23) and determine the confidence bounds using the $\mathrm{CDF}$.

(b) Case: the product of $\phi^{\prime} A^{\prime} / A$ or $\phi^{\prime} A^{\prime} / A+1 /(2 \rho)$

1. Approximate mean values and variances using the moment generating function for every harmonic and use only the harmonics.

2. Combine the different mean values $\mu_{p}\left(\Omega_{k}\right)$ and variances $\sigma_{p}^{2}\left(\Omega_{k}\right)$ using (39), but with weighting $\Omega_{k}$ instead of $\sqrt{\Omega_{k}}$ to determine $\mu_{m p}$ and its variance $\sigma_{m p}^{2}$ using (40).

3. Calculate the overall diffusivity

$$
\chi=\frac{3}{4} \frac{1}{\mu_{m p}} .
$$

4. The corresponding confidence bounds are calculated by substituting $\mu_{m p}$, $\sigma_{m p}^{2}$, and $\Omega=1$ in (27) and determine the CDF, which is acquired by numerically integrating (27).

In practice, the diffusivity is often rescaled in terms of SI units. The confidence bounds can also be rescaled accordingly.

\section{Conclusions and Discussion}

In this paper, the effect of uncertainty on the estimates of the diffusivity is studied. The properties of ECE-measurements and of the Fourier transform are used to determine the noise distribution of the Fourier coefficients. Based on this bivariate distribution, it is shown that amplitude, phase, and their derivatives are well approximated by Gaussian distributions for large SNRs. Then, the diffusion coefficient is distributed according to the inverse non-central chi-squared distribution when the relationships in (1) and (2) are used. Its analytically derived CDF is used to determine the confidence bounds on the diffusivity, i.e. its accuracy.

Based on the distributions of $\chi$ and its confidence bounds different methods to combine harmonics, amplitude, and phase are compared including the cases with 
damping. The (generalized) weighted mean gives the resulting Gaussian distribution with the smallest variance. In case of the product an approximation for the product of phase and amplitude derivatives are necessary introducing some errors. It outperforms other methods commonly used to combine different harmonics, amplitude, and phase in fusion. Moreover, it gives an estimate of the variance, which is necessary to determine the confidence bounds on $\chi$. In addition, the direct diffusivity estimate based on the ASDEX Upgrade data of the first harmonic already shows a near Gaussian distribution, which is an indication that it is close to the optimal solution (in case of . However, many issues arise from using amplitude and phase to determine the diffusivity. The amplitude and phase are correlated quantities in contrast to the real and imaginary parts of the Fourier coefficients. Taking the squared reciprocal of Gaussian distributed variables always gives a distribution with a long tail and hence a high upper uncertainty. This effect becomes much larger when the variances increase. For example, this is important when the time evolution of the diffusion coefficient needs to be studied, because a reduced number of periods leads to a higher variance. Therefore, relationships based on inverting Gaussian distributed variables as the phase and amplitude should be avoided to arrive at an optimal estimate of $\chi$. However, this requires implicit estimation schemes to determine the diffusivity, which often results in non-convex optimization problems.

\section{Acknowledgments}

The authors are grateful for the useful comments and suggestions made by Dr. François Ryter.

This project has received funding from the European Union's Horizon 2020 research and innovation programme under grant agreement number 633053. The views and opinions expressed herein do not necessarily reflect those of the European Commission. This work is also supported by NWO-RFBR Centre-of-Excellence on Fusion Physics and Technology (Grant nr. 047.018.002). This work was also partly supported by the Fund for Scientific Research (FWO-Vlaanderen), by the Flemish Government (Methusalem), and by the Belgian Government through the Interuniversity Poles of Attraction (IAP VII/4 Dysco) Program. The first author is an international research fellow of the Japan Society for the Promotion of Science and hence wishes to express his gratitude to the JSPS for making this research possible.

\section{Appendix A. Distributions of Amplitude and Phase}

Here, the PDFs and CDFs of amplitude and phase are introduced, which are necessary to calculate their confidence bounds. These PDFs and CDFs are well known in the literature $[41,45]$.

The PDF of the amplitude can be found by integrating (12) over the phase

$$
f_{A}(A)=\int_{0}^{2 \pi} f_{A \phi}(A, \phi) d \phi,
$$

which results in

$$
f_{A}(A)=\frac{A}{2 \pi \sigma_{F}^{2}} \exp \left(-\frac{A^{2}+M^{2}}{2 \sigma_{F}^{2}}\right) \int_{0}^{2 \pi} \exp \left(\frac{A M \cos (\theta-\phi)}{\sigma_{F}^{2}}\right) d \phi .
$$


The resulting amplitude distribution is given by

$$
f_{A}(A)=\frac{A}{\sigma_{F}^{2}} \exp \left(-\frac{A^{2}+M^{2}}{2 \sigma_{F}^{2}}\right) I_{0}\left(\frac{A M}{\sigma_{F}^{2}}\right),
$$

where $I_{0}$ denotes the modified Bessel function of the first kind of zero order. The confidence bounds are calculated using the CDF. This CDF $F_{A}(R)$ can be found by integrating the $\mathrm{PDF} f_{A}(A)$ from 0 to $R$,

$$
F_{A}(R)=\int_{0}^{R} f_{A}(A) d A=1-Q_{1}\left(\frac{M}{\sigma_{F}}, \frac{R}{\sigma_{F}}\right),
$$

where $Q_{1}$ denotes the Marcum Q-function [41]. The Rician distribution is nonsymmetric, which means that two confidence bounds need to be determined separately in terms of a confidence $\mathfrak{p}$, e.g. $\mathfrak{p}=0.95$. A central confidence interval is constructed such that the lower bound is defined as $F_{A}\left(A_{\min }\right)=(1-\mathfrak{p}) / 2$ and the upper bound as $F_{A}\left(A_{\max }\right)=(1+\mathfrak{p}) / 2$. These bounds are calculated by inverting (A.4) numerically (see Appendix B).

The PDF of the phase $\phi$ is derived by integrating the CCND in polar coordinates (12) over $A$

$$
f_{\phi}(\phi)=\int_{0}^{\infty} f_{A \phi}(A, \phi) d A .
$$

This integral can be solved using the substitution $\kappa=A-M \cos (\theta-\phi)$ [45] resulting in

$$
\begin{aligned}
& f_{\phi}(\phi)=\exp \left(-\frac{M^{2}}{2 \sigma^{2}}\right)\left[\frac{1}{2 \pi}+\frac{M \cos (\theta-\phi)}{2 \sigma_{F} \sqrt{2 \pi}} .\right. \\
& \left.\exp \left(\frac{M^{2} \cos ^{2}(\theta-\phi)}{2 \sigma_{F}^{2}}\right) \operatorname{erfc}\left(\frac{M \cos (\theta-\phi)}{\sigma_{F} \sqrt{2}}\right)\right],
\end{aligned}
$$

This distribution is sometimes referred to as the Rician phase distribution [45]. Again, we are interested in the confidence bounds, which can be calculated via the CDF

$$
F_{\phi}(\Phi)=\int_{-\pi}^{\Phi} f_{\phi}(\phi) d \phi
$$

where $-\pi<\Phi \leqslant \pi$. This integral does not have a closed form solution, but can be approximated numerically, which is explained next.

\section{Appendix B. Constructing confidence bounds numerically}

The Cumulative Density Functions of (27) necessary to calculate the confidence bounds on $\chi$ when using the product $A^{\prime} / A \phi^{\prime}$ and the CDF of the phase (A.7) necessary to calculate the confidence bounds on the phase do not have closed-form solutions. Therefore, here is explained how the confidence bounds can be calculated numerically exploiting properties of the integrals. The CDF of $h\left(\chi\left(\mu_{p}\right)\right)$ defined in (27) is given by the integral

$$
H(X)=\int_{-\infty}^{X} \frac{3}{4} \frac{\Omega}{\chi^{2}} \frac{1}{\sqrt{2 \pi \sigma_{p}^{2}}} \exp \left(-\frac{\left(\frac{3 \Omega}{4 \chi}-\mu_{p}\right)^{2}}{2 \sigma_{p}^{2}}\right) d \chi .
$$


This integral needs to be evaluated numerically and then needs to be inverted. However, integrating from minus infinity is impractical. Therefore, this integral is split into two parts

$$
H(X)=\int_{-\infty}^{0} h\left(\chi\left(\mu_{p}\right)\right) d \chi+\int_{0}^{X} h\left(\chi\left(\mu_{p}\right)\right) d \chi .
$$

where the first term can be evaluated analytically giving

$$
H_{0}=\int_{-\infty}^{0} h\left(\chi\left(\mu_{p}\right)\right) d \chi=\frac{1}{2}\left(1-\operatorname{erf}\left(\frac{\mu_{p}}{\sqrt{2} \sigma_{p}}\right)\right)
$$

If the diffusion coefficient is assumed to be always positive, this term should be always smaller than the lower confidence bound $H_{0}<(1-\mathfrak{p}) / 2$ and certainly smaller than the upper confidence bound $H_{0}<(1+\mathfrak{p}) / 2$. This is of course not guaranteed numerically as the product $A^{\prime} / A \phi^{\prime}$ can become negative and hence also its confidence bounds. However, both special cases can be easily evaluated and if the lower bound is negative we have decided to simply set it to zero $(\chi>0)$. If both bounds are negative there is clearly something wrong as such that case should be fully ignored. Hence, to calculate proper confidence bounds it suffices to numerically integrate

$$
H(X)=H_{0}+\int_{0}^{X} \frac{3}{4} \frac{\Omega}{\chi^{2}} \frac{1}{\sqrt{2 \pi \sigma_{p}^{2}}} \exp \left(-\frac{\left(\frac{3 \Omega}{4 \chi}-\mu_{p}\right)^{2}}{2 \sigma_{p}^{2}}\right) d \chi .
$$

where 0 has been replaced by a very small number and verifying that $H_{0}<(1-\mathfrak{p}) / 2$ and $H_{0}<(1+\mathfrak{p}) / 2$.

Calculating the CDF of the phase $F_{\phi}(\Phi)$ is straightforward as the distribution $f_{\phi}(\phi)$ is symmetric around the mean $\theta$ and its integration interval is already bounded $-\pi<\Phi \leqslant \pi$.

CDFs have been found for $H(X)$ and $F_{\phi}(\Phi)$ in terms of a numerical integration from 0 to $X$ and $-\pi$ to $\Phi$, respectively. However, the question is how to invert these relationships to find $X_{\text {bnd }} H\left(X_{\text {bnd }}\right)=(1 \pm \mathfrak{p}) / 2$.

The CDF is a (non-decreasing) monotonic function and is bounded in the domain 0 to 1 . This means that by shifting it $H_{\text {shift }}(X)=H(X)-(1-\mathfrak{p}) / 2$ gives exactly one zero, which can be found numerically using various techniques [53]. This techniques is also applied to find the confidence bounds for (A.4) and (20). In case of (A.4) and (20) we found it useful to use the property $H(|X|)=|H(|X|)|$, which allows also the use negative $X$ such that an unconstrained algorithm can be used.

\section{Appendix C. Analytic distributions of $A^{\prime} / A$ for uncorrelated $\Theta\left(\rho_{1}\right)$ and $\Theta\left(\rho_{2}\right)$}

The joint distribution function of $A_{1}$ and $A_{2}$ is derived here based on (9). Therefore, it is assumed that the amplitudes on two different spatial locations are independent, hence the joint distribution is determined by the product of the two Gaussian approximations of the amplitude

$$
f\left(A_{1}, A_{2}\right)=\frac{1}{2 \pi \sigma_{A_{1}} \sigma_{A_{2}}} \exp \left(-\frac{\left(A_{1}-M_{1}\right)^{2}}{2 \sigma_{A_{1}}^{2}}-\frac{\left(A_{2}-M_{2}\right)^{2}}{2 \sigma_{A_{2}}^{2}}\right) .
$$


Next, the change of variables $y_{1}=\ln \left(A_{2} / A_{1}\right) / \Delta \rho$ and $y_{2}=A_{2}$ is introduced. The joint distribution in terms of $y_{1}$ and $y_{2}$ is described using conservation of area [31]

$$
g\left(y_{1}, y_{2}\right)=\frac{y_{2} \Delta \rho}{2 \pi \sigma_{d}\left(y_{1}\right) \sigma_{A_{2}}} \exp \left(-\frac{\left(y_{2}-M_{d}\left(y_{1}\right)\right)^{2}}{2 \sigma_{d}^{2}\left(y_{1}\right)}-\frac{\left(y_{2}-M_{2}\right)^{2}}{2 \sigma_{A_{2}}^{2}}\right)
$$

where $\sigma_{d}=\sigma_{A_{1}} / d$, and $M_{d}=M_{1} / d$, with $d\left(y_{1}=A^{\prime} / A\right)=\exp \left(-\Delta \rho y_{1}\right)$. This distribution is used to find the distribution of $y_{1}=A^{\prime} / A$, by integrating (C.2) over $y_{2}$, i.e. $g\left(y_{1}\right)=\int_{-\infty}^{\infty} g\left(y_{1}, y_{2}\right) d y_{2}$. This results in

$$
g\left(\frac{A^{\prime}}{A}\right)=\frac{\Delta \rho\left(M_{2} \sigma_{d}^{2}+M_{d} \sigma_{A_{2}}^{2}\right)}{\sqrt{2 \pi}\left(\sigma_{d}^{2}+\sigma_{A_{2}}^{2}\right)^{3 / 2}} \exp \left(-\frac{1}{2} \frac{\left(M_{2}-M_{d}\right)^{2}}{\sigma_{d}^{2}+\sigma_{A_{2}}^{2}}\right) .
$$

This distribution is rather complicated, but can also often be approximated by a Gaussian distribution for reasonable SNRs.

\section{References}

[1] J. P. Freidberg, Plasma Physics and Fusion Energy. Cambridge University Press, 2007.

[2] J. Wesson, Tokamaks. OUP Oxford, 2011, vol. 149.

[3] R. Balescu, Aspects of anomalous transport in plasmas. Taylor \& Francis, 2005.

[4] N. J. Lopes Cardozo, "Perturbative transport studies in fusion plasmas," Plasma Phys. Control. Fusion, vol. 37, p. 799, 1995.

[5] F. Ryter, R. Dux, P. Mantica, and T. Tala, "Perturbative studies of transport phenomena in fusion devices," Plasma Phys. Control. Fusion, vol. 52, p. 124043, 2010.

[6] F. Ryter, G. Tardini, F. D. Luca, H.-U. Fahrbach, F. Imbeaux, A. Jacchia, K. Kirov, F. Leuterer, P. Mantica, A. Peeters, G. Pereverzev, W. Suttrop, and the ASDEX Upgrade Team, "Electron heat transport in ASDEX Upgrade: experiment and modelling," Nuclear Fusion, vol. 43, no. 11, p. 1396, 2003.

[7] S. Inagaki, T. Tokuzawa, K. Itoh, K. Ida, S.-I. Itoh, N. Tamura, S. Sakakibara, N. Kasuya, A. Fujisawa, S. Kubo, T. Shimozuma, T. Ido, S. Nishimura, H. Arakawa, T. Kobayashi, K. Tanaka, Y. Nagayama, K. Kawahata, S. Sudo, H. Yamada, and A. Komori, "Observation of long-distance radial correlation in toroidal plasma turbulence," Phys. Rev. Lett., vol. 107, p. 115001, Sep 2011. [Online]. Available: http://link.aps.org/doi/10.1103/PhysRevLett.107.115001

[8] T. Tala, K.-D. Zastrow, J. Ferreira, P. Mantica, V. Naulin, A. G. Peeters, G. Tardini, M. Brix, G. Corrigan, C. Giroud, and D. Strintzi, "Evidence of inward toroidal momentum convection in the JET Tokamak," Phys. Rev. Lett., vol. 102, p. 075001, Feb 2009. [Online]. Available: http://link.aps.org/doi/10.1103/PhysRevLett.102.075001

[9] G. Tardini, J. Ferreira, P. Mantica, A. Peeters, T. Tala, K. Zastrow, M. Brix, C. Giroud, G. Pereverzev, and J.-E. contributors, "Angular momentum studies with nbi modulation in jet," Nuclear Fusion, vol. 49, no. 8, p. 085010, 2009. [Online]. Available: http://stacks.iop.org/0029-5515/49/i=8/a $=085010$

[10] P. Mantica, T. Tala, J. Ferreira, A. Peeters, A. Salmi, D. Strintzi, J. Weiland, M. Brix, C. Giroud, G. Corrigan et al., "Perturbative studies of toroidal momentum transport using neutral beam injection modulation in the Joint European Torus: Experimental results, analysis methodology, and first principles modeling," Physics of Plasmas (1994-present), vol. 17, no. 9, p. 092505, 2010.

[11] P. Mantica, D. Strintzi, T. Tala, C. Giroud, T. Johnson, H. Leggate, E. Lerche, T. Loarer, A. Peeters, A. Salmi et al., "Experimental study of the ion critical-gradient length and stiffness level and the impact of rotation in the JET tokamak," Physical review letters, vol. 102, no. 17, p. 175002, 2009.

[12] B. Zurro, E. Hollmann, A. Baciero, M. Ochando, F. Medina, K. McCarthy, E. Blanco, E. de la Cal, D. Carralero, M. Pedrosa et al., "Transport studies using laser blow-off injection of low-Z trace impurities injected into the TJ-II stellarator," Nuclear Fusion, vol. 51, no. 6, p. 063015, 2011.

[13] Z. Hangyu, C. Zhengying, M. Shigeru, F. Bingzhong, G. Motoshi, S. Ping, F. Beibin, C. Xuewu, L. Ping, Y. Qingwei, and D. Xuru, "Spectral analysis in euv range for study of core impurity 
Estimation of the thermal diffusion coefficient in fusion plasmas

behavior in hl-2a," Plasma Science and Technology, vol. 16, no. 2, p. 89, 2014. [Online]. Available: http://stacks.iop.org/1009-0630/16/i=2/a=01

[14] N. Howard, M. Greenwald, D. Mikkelsen, M. Reinke, A. White, D. Ernst, Y. Podpaly, and J. Candy, "Quantitative comparison of experimental impurity transport with nonlinear gyrokinetic simulation in an alcator c-mod l-mode plasma," Nuclear Fusion, vol. 52, no. 6, p. $063002,2012$.

[15] H. J. Hartfuss, T. Geist et al., "Heterodyne methods in millimetre wave plasma diagnostics with applications to ECE, interferometry and reflectometry," Plasma Phys. Control. Fusion, vol. 39, pp. 1693-1769, 1997.

[16] K. W. Gentle, "Dependence of heat pulse propagation on transport mechanisms: Consequences of nonconstant transport coefficients," Physics of Fluids, vol. 31, p. 1105, 1988.

[17] A. Jacchia, P. Mantica, F. De Luca, and G. Gorini, "Determination of diffusive and nondiffusive transport in modulation experiments in plasmas," Physics of Fluids B, vol. 3, no. 11, pp. 3033-3040, 1991.

[18] D. J. Gambier, M. P. Evrard, J. Adam, A. Becoulet, S. Corti, P. Hennequin, J. Jacquinot, D. F. H. Start, K. Thomsen, B. J. D. Tubbing et al., "ICRF power deposition profile and determination of the electron thermal diffusivity by modulation experiments in JET," Nuclear Fusion, vol. 30, no. 1, p. 23, 1990.

[19] N. J. Lopes Cardozo and J. C. M. de Haas, "Heat pulse analysis in JET limiter and x-point plasmas," Nuclear Fusion, vol. 30, no. 3, p. 521, 1990.

[20] G. M. D. Hogeweij, J. O'Rourke, and A. C. C. Sips, "Evidence of coupling of thermal and particle transport from heat and density pulse measurements in JET," Plasma Phys. Control. Fusion, vol. 33, no. 3, p. 189, 1991.

[21] P. Mantica, M. Peters, F. De Luca, A. De Lauri, G. Gorini, G. M. D. Hogeweij, A. Jacchia, and N. J. Lopes Cardozo, "Radial profile and q dependence of electron heat diffusion measured with ech modulation in RTP," Nuclear Fusion, vol. 36, no. 10, p. 1317, 1996.

[22] P. Mantica, G. Gorini, G. M. D. Hogeweij, N. J. Lopes Cardozo, and A. M. R. Schilham, "Heat convection and transport barriers in low-magnetic-shear rijnhuizen tokamak project plasmas," Phys. Rev. Lett., vol. 85, no. 21, pp. 4534-4537, 2000.

[23] F. Ryter, C. Angioni, A. G. Peeters, F. Leuterer, H.-U. Fahrbach, and W. Suttrop, "Experimental study of trapped-electron-mode properties in tokamaks: Threshold and stabilization by collisions," Phys. Rev. Lett., vol. 95, p. 085001, Aug 2005.

[24] J. DeBoo, S. Cirant, T. Luce, A. Manini, C. Petty, F. Ryter, M. Austin, D. Baker, K. Gentle, C. Greenfield, J. Kinsey, and G. Staebler, "Search for a critical electron temperature gradient in DIII-D L-mode discharges," Nuclear Fusion, vol. 45, no. 6, p. 494, 2005.

[25] J. DeBoo, C. Petty, A. White, K. Burrell, E. Doyle, J. Hillesheim, C. Holland, G. McKee, T. Rhodes, L. Schmitz et al., "Electron profile stiffness and critical gradient studies," Physics of Plasmas, vol. 19, p. 082518, 2012.

[26] E. D. Fredrickson, M. E. Austin, R. Groebner, J. Manickam, B. Rice, G. Schmidt, and R. Snider, "Heat pulse propagation studies on DIII-D and the Tokamak Fusion Test Reactor," Physics of Plasmas, vol. 7, p. 5051, 2000.

[27] L. Giannone, V. Erckmann, U. Gasparino, H. J. Hartfuss, G. Kuhner, H. Maassberg, U. Stroth, and M. Tutter, "Electron thermal conductivity from heat wave propagation in Wendelstein 7-AS," Nuclear Fusion, vol. 32, no. 11, p. 1985, 1992.

[28] S. Inagaki, H. Takenaga, K. Ida, A. Isayama, N. Tamura, T. Takizuka, T. Shimozuma, Y. Kamada, S. Kubo, Y. Miura et al., "Comparison of transient electron heat transport in LHD helical and JT-60U tokamak plasmas," Nuclear Fusion, vol. 46, no. 1, p. 133, 2006.

[29] G. W. Spakman, G. M. D. Hogeweij, R. Jaspers, F. C. Schüller, E. Westerhof, J. E. Boom, I. G. J. Classen, E. Delabie, C. Domier, A. J. H. Donné et al., "Heat pulse propagation studies around magnetic islands induced by the dynamic ergodic divertor in textor," Nuclear Fusion, vol. 48, p. 115005, 2008.

[30] M. Hölzl, S. Günter, I. Classen, Q. Yu, E. Delabie et al., "Determination of the heat diffusion anisotropy by comparing measured and simulated electron temperature profiles across magnetic islands," Nuclear Fusion, vol. 49, no. 11, p. 115009, 2009.

[31] B. Martin, Statistics for Physical Sciences: An Introduction. Waltham MA: Academic Press, 2012.

[32] Oliveira, A. and Oliveira, Teresa A and Seijas-Macias, A, "The influence of ratios and combined ratios on the distribution of the product of two independent gaussian random variables," in Proceedings of the 59th World Statistics Congress of the International Statistical Institute, 2013.

[33] N. L. Cardozo, B. Tubbing, F. Tibone, and A. Taroni, "Heat pulse propagation: Diffusive models 
checked against full transport calculations," Nuclear Fusion, vol. 28, no. 7, p. 1173, 1988.

[34] E. Fredrickson, J. Callen, K. McGuire, J. Bell, R. Colchin, P. Efthimion, K. Hill, R. Izzo, D. Mikkelsen, D. Monticello et al., "Heat pulse propagation studies in tftr," Nuclear Fusion, vol. 26 , no. 7 , p. 849,1986 .

[35] M. Soler and J. Callen, "On measuring the electron heat diffusion coefficient in atokamak from sawtooth oscillation observations," Nuclear Fusion, vol. 19, no. 6, p. 703, 1979.

[36] M. van Berkel et al., "Explicit approximations to estimate the perturbative diffusivity in the presence of convectivity and damping (part a): slab-geometry approximations," submitted to Physics of Plasmas, vol. -, pp. -, 2014.

[37] P. Billingsley, Probability and measure. John Wiley and Sons, New York, 1995.

[38] R. C. Emerson, "First probability densities for receivers with square law detectors," Journal of Applied Physics, vol. 24, no. 9, pp. 1168-1176, 1953.

[39] S. Sattler and H. J. Hartfuss, "Intensity interferometry for measurement of electron temperature fluctuations in fusion plasmas," Plasma Phys. Control. Fusion, vol. 35, no. 9, p. 1285, 1993.

[40] N. R. Goodman, "Statistical analysis based on a certain multivariate complex Gaussian distribution (an introduction)," The Annals of Mathematical Statistics, vol. 34, no. 1, pp. $152-177,1963$.

[41] A. D. Whalen, Detection of signals in noise. Academic Press New York, 1971, vol. 6.

[42] D. P. Kroese, T. Taimre, and Z. I. Botev, Handbook of Monte Carlo Methods. John Wiley and Sons, 2011, vol. 706 .

[43] R. Pintelon and J. Schoukens, System Identification: A Frequency Domain Approach. WileyIEEE Press, Hoboken (NJ), 2012.

[44] D. R. Brillinger, Time series: data analysis and theory. SIAM Philadelphia (PA), 2001.

[45] M. K. Simon and M.-S. Alouini, Digital communication over fading channels. WileyInterscience, 2005, vol. 95.

[46] A. Seijas-Macias and A. Oliveira, "An approach to distribution of the product of two normal variables," Discussiones Mathematicae Probability and Statistics, vol. 32, pp. 87-99, 2012.

[47] L. A. Aroian, V. S. Taneja, and L. W. Cornwell, "Mathematical forms of the distribution of the product of two normal variables," Communications in Statistics-Theory and Methods, vol. 7, no. 2 , pp. $165-172,1978$.

[48] C. C. Craig, "On the frequency function of xy," The Annals of Mathematical Statistics, vol. 7, no. 1 , pp. 1-15, 1936.

[49] R. Ware and F. Lad, "Approximating the distribution for sums of products of normal variables," University of Canterbury, England, Tech. Rep. UCDMS, vol. 15, p. 2003, 2003.

[50] P. Mantica, F. Ryter, C. Capuano, H. U. Fahrbach, F. Leuterer, W. Suttrop, J. Weiland, and ASDEX-Upgrade Team, "Investigation of electron heat pinch in ASDEX-Upgrade," Plasma Phys. Control. Fusion, vol. 48, no. 3, p. 385, 2006.

[51] P. Mantica and F. Ryter, "Perturbative studies of turbulent transport in fusion plasmas," Comptes Rendus Physique, vol. 7, no. 6, pp. 634-649, 2006.

[52] M. G. Cox, C. Eiø, G. Mana, and F. Pennecchi, "The generalized weighted mean of correlated quantities," Metrologia, vol. 43, no. 4, p. S268, 2006.

[53] G. E. Forsythe, C. B. Moler, and M. A. Malcolm, Computer methods for mathematical computations. Prentice-Hall, 1977. 